\title{
Neon, sulphur, and argon abundances of planetary nebulae in the sub-solar metallicity Galactic anti-centre
}

\author{
G. J. S. Pagomenos ${ }^{1}$, J. Bernard-Salas ${ }^{1}$, and S. R. Pottasch ${ }^{2}$ \\ ${ }^{1}$ School of Physical Sciences, The Open University, Milton Keynes MK7 6AA, UK \\ e-mail: george . pagomenos@open.ac.uk \\ 2 Kapteyn Astronomical Institute, PO Box 800, 9700 AV Groningen, The Netherlands
}

Received 23 March 2017 / Accepted 16 December 2017

\begin{abstract}
Context. Spectra of planetary nebulae show numerous fine structure emission lines from ionic species, enabling us to study the overall abundances of the nebular material that is ejected into the interstellar medium. The abundances derived from planetary nebula emission show the presence of a metallicity gradient within the disk of the Milky Way up to Galactocentric distances of $\sim 10 \mathrm{kpc}$, which are consistent with findings from studies of different types of sources, including H II regions and young B-type stars. The radial dependence of these abundances further from the Galactic centre is in dispute.

Aims. We aim to derive the abundances of neon, sulphur and argon from a sample of planetary nebulae towards the Galactic anticentre, which represent the abundances of the clouds from which they were formed, as they remain unchanged throughout the course of stellar evolution. We then aim to compare these values with similarly analysed data from elsewhere in the Milky Way in order to observe whether the abundance gradient continues in the outskirts of our Galaxy.

Methods. We have observed 23 planetary nebulae at Galactocentric distances of 8-21 kpc with Spitzer IRS. The abundances were calculated from infrared emission lines, for which we observed the main ionisation states of neon, sulphur, and argon, which are little affected by extinction and uncertainties in temperature measurements or fluctuations within the planetary nebula. We have complemented these observations with others from optical studies in the literature, in order to reduce or avoid the need for ionisation correction factors in abundance calculations.

Results. The overall abundances of our sample of planetary nebulae in the Galactic anti-centre are lower than those in the solar neighbourhood. The abundances of neon, sulphur, and argon from these stars are consistent with a metallicity gradient from the solar neighbourhood up to Galactocentric distances of $\sim 20 \mathrm{kpc}$, albeit with varying degrees of dispersion within the data.
\end{abstract}

Key words. stars: late-type - stars: abundances - planetary nebulae - general - dust, extinction - infrared: ISM - infrared: stars

\section{Introduction}

As Sun-like stars (of $\sim 0.8-8 M_{\odot}$ ) evolve, they eventually become planetary nebulae $(\mathrm{PNe})$. In this evolutionary phase, the star has lost enough of its convective envelope through stellar winds to expose its inner, hotter regions, causing this ejected material to become ionised. We can determine the ionic and elemental abundances of this PN ejecta by analysing the strong forbidden emission lines of the ions in the stellar spectrum.

Much of the observable PN emission comes from ionisation states of $\alpha$-process elements, such as neon, sulphur, and argon. The main stages of ionisation for these three elements are observed at mid-infrared wavelengths. While the abundances of elements such as helium and carbon change significantly throughout the course of stellar evolution, those of neon, sulphur, and argon are unchanged (e.g. Marigo et al. 2003), making them useful probes of metallicity at the epoch of stellar formation. Abundance studies carried out at optical wavelengths commonly use oxygen as a metric for metallicity, as the observed emission lines of $\mathrm{O}^{+}$and $\mathrm{O}^{2+}$ are always strong. However, during the evolution of these low- to intermediate-mass stars, the abundances of oxygen are known to change. This particularly occurs within the asymptotic giant branch (AGB) phase, in which the third dredge-up brings helium, carbon and a small amount of oxygen to the outer envelope of the star. For stars with $M \gtrsim 4 M_{\odot}$, some oxygen will also be destroyed by hot bottom burning (e.g. Karakas \& Lattanzio 2014; Delgado-Inglada et al. 2015). An empirical study by Delgado-Inglada et al. (2015) has also shown that oxygen enrichment can occur in Galactic PNe with carbonaceous dust. It has been proposed by García-Hernández et al. (2016) that this can be explained by diffusive convective overshooting processes, in which core mixing is extended beyond the Schwarzschild boundary of main sequence stars (Böhm-Vitense 1958; Herwig et al. 1997), producing significant increases in oxygen abundances around sub-solar and solar metallicities (Marigo 2001; Pignatari et al. 2016).

While most abundances from observational studies have been measured using optical spectra, there are some advantages to analysing PNe using infrared spectra. These are described in several studies (e.g. Rubin et al. 1988; Pottasch \& Beintema 1999; Bernard-Salas 2003), but can be summarised as follows: extinction corrections are greatly reduced at IR wavelengths compared to those in the optical and ultraviolet regions; many ionic emission lines are observable for $\mathrm{Ne}, \mathrm{S}$ and $\mathrm{Ar}$ within this wavelength range, and hence the need for ionisation correction factors (ICFs) in calculating elemental abundances is reduced; as these IR lines also originate from energy levels close to the ground state, both the uncertainties in the electron temperatures of any ion measured at IR wavelengths and temperature fluctuations within the PN can have little effect on the 
overall abundances. For this study in particular, the extinction corrections are reduced further as we have analysed the Galactic anti-centre, a region with much less extinction than the bulge (e.g. Pottasch \& Bernard-Salas 2015).

The presence of the Galactic metallicity gradient was made clear in a sample of H II regions by Shaver et al. (1983) for nitrogen, oxygen, sulphur, and argon. Since then, it has been further studied not only in H II regions (e.g. Martín-Hernández et al. 2002; Esteban et al. 2017; Fernández-Martín et al. 2017) but also in PNe (e.g. Maciel \& Quireza 1999; Pottasch \& Bernard-Salas 2006; Maciel et al. 2015), young B-type stars (e.g. Fitzsimmons et al. 1992; Rolleston et al. 2000), Cepheid variables (e.g. Andrievsky et al. 2002a,b,c; Luck et al. 2003; Lemasle et al. 2013; Genovali et al. 2015), open clusters (e.g. Friel 1995) and young stars (e.g. Magrini et al. 2017).

While the presence of the metallicity gradient in the Galactic disk is agreed upon over Galactocentric distances $\left(R_{g}\right)$ in the range 4-10 kpc, its continuation towards the anti-centre is debated. Studies of H II regions (Esteban et al. 2017; Fernández-Martín et al. 2017) and B-type stars (Smartt 2000) have found that there is little variation in the gradient far from the Galactic centre, yet a study of Cepheid variables from Andrievsky et al. (2002c) showed the gradient flattening with $R_{g}$. Samples of PNe have also been previously analysed in the anti-centre with conflicting results. Costa et al. (2004) showed that the oxygen abundances of a group of PNe, 8-15 kpc away from the Galactic centre, did not directly follow the gradient but instead flattened beyond $10 \mathrm{kpc}$. This has also been observed in the nearby spiral galaxies M31, M33, M81 and NGC 300 (Magrini et al. 2016). However, the sample of Henry et al. (2010) suggested that the gradient steepened beyond this distance.

Chemical evolution models of the Milky Way have predicted that the radial abundance gradient will flatten over time due to several factors, such as the death of massive stars, which causes the metallicity to increase over time (e.g. Minchev et al. 2013), and radial migration (e.g. Minchev et al. 2012, 2014; Vera-Ciro et al. 2014; Kubryk et al. 2015), in which the angular momentum from stars is redistributed, leading to the movement of stars from the Galactic disk and hence contributing to a flattening radial metallicity gradient within the disk (e.g. Sellwood \& Binney 2002).

Investigations into the time evolution of the radial metallicity gradient have given varying results, with several studies of PNe finding an overall steepening with time (e.g. Maciel \& Quireza 1999; Chiappini et al. 2001; Stanghellini \& Haywood 2010; Kubryk et al. 2015), suggesting that the Galactic disk formed slowly (Chiappini et al. 1997). However, Maciel et al. (2003) showed the gradient flattening over time. Studies of open clusters and field stars have also given varying conclusions on this matter (Anders et al. 2017).

In this paper, we have derived the abundances of neon, sulphur, and argon in a sample of $23 \mathrm{PNe}$ located towards the Galactic anti-centre using IR data in order to study the metallicity gradient beyond $10 \mathrm{kpc}$, and compared them to other IR spectroscopic samples from the Milky Way that were analysed in the same way.

The layout of this paper is as follows: In Sect. 2 we discuss the source selection and the basic data reduction and extraction methods. In Sect. 3 we explain the methods used to calculate flux, intensity and abundance values as well as Galactocentric distances. The implications of these data on the metallicity gradient in the further regions of the Milky Way are considered in Sect. 4. Finally, in Sect. 5 we present our conclusions and summarise our main results.

\section{Data}

\subsection{Observations}

The observations were made with the Infrared Spectrograph on board the Spitzer Space Telescope (Spitzer IRS; Werner et al. 2004; Houck et al. 2004) through GTO programme 40035 (PI: J. Bernard-Salas). The observations were carried out between December 2007 and December 2008 with the staring mode of the IRS using the short-low (SL), short-high (SH) and longhigh (LH) modules, each allowing for simultaneous observations from two nod positions, at $1 / 3$ and $2 / 3$ of the way along the observing slit. These produced spectra with resolutions of $R \sim 60-127$ in the range 5.2-14.5 $\mu \mathrm{m}$, and of $R \sim 600$ in the range 9.9-36.4 $\mu \mathrm{m}$.

The sources are listed in Table A.1. These were chosen according to the following two criteria: (a) the sources were located in the direction of the anti-centre $\left(l=120-240^{\circ}, b=\right.$ $0^{\circ} \pm 20^{\circ}$ ); (b) the physical sizes of the PNe were generally small enough to fit in the widest observing slit of Spitzer IRS $\left(\mathrm{LH}, 11.1^{\prime \prime} \times 22.3^{\prime \prime}\right)$, thus minimising the aperture corrections required to account for the different slit sizes.

\subsection{Data reduction and extraction}

The basic calibration data $(b c d)$ image files obtained from the Spitzer IRS were processed through the Spitzer Science Centre (SSC) pipeline, version S18.18, then reduced and analysed through the Spectroscopic Modelling Analysis and Reduction Tool (SMART; Higdon et al. 2004). Rogue pixels were removed using the IRSCLEAN ${ }^{1}$ package.

The methods of spectral extraction were based on the diameters of the sources (see Table A.2). For the low-resolution SL module, sources with diameters $\leqslant 3^{\prime \prime}$ were extracted using the Advanced Optimal Extraction (AdOpt) package (Lebouteiller et al. 2010). This method weights each pixel based on their signal-to-noise ratios and is better suited to smaller sources. Those with diameters in the range $3^{\prime \prime}-8^{\prime \prime}$ were extracted using tapered column extractions, as the SL slit is not wide enough $\left(3.6^{\prime \prime} \times 57^{\prime \prime}\right)$ to detect all the flux in partially extended sources. The four PNe with diameters $\geqslant 10^{\prime \prime}$ were extracted using fixed column extractions as the FWHM of their emission was beyond the point spread function of the objects by factors up to approximately three. For the high-resolution $\mathrm{SH}$ and $\mathrm{LH}$ modules, full aperture extractions were used in each case; these weight all pixels equally from the aperture, allowing for most of the flux to be obtained from more extended sources if they covered an area larger than the observing slits. The flux values of a PN with a physical size bordering on two of these ranges do not change significantly $(\lesssim 10 \%)$.

Most of the PNe in our sample were chosen such that all of the source flux would fall within the LH module. In fact, 15 of our 23 targets also have a physical diameter of $\leqslant 5^{\prime \prime}$, so in these cases most of the flux would also be detected by the smaller and narrower SH module $\left(4.7^{\prime \prime} \times 11.3^{\prime \prime}\right)$. Some of the source flux might still not be detected by the SL and SH modules, resulting in jumps in the baseline continuum. To account for this, we scale the SL and SH flux values by matching the continua in the overlapping wavelength regions. The scale factors are listed in

\footnotetext{
1 Available from the SSC website: http://ssc. spitzer. caltech. edu.
} 
G. J. S. Pagomenos et al.: Ne, S, and Ar abundances in Galactic anti-centre PNe

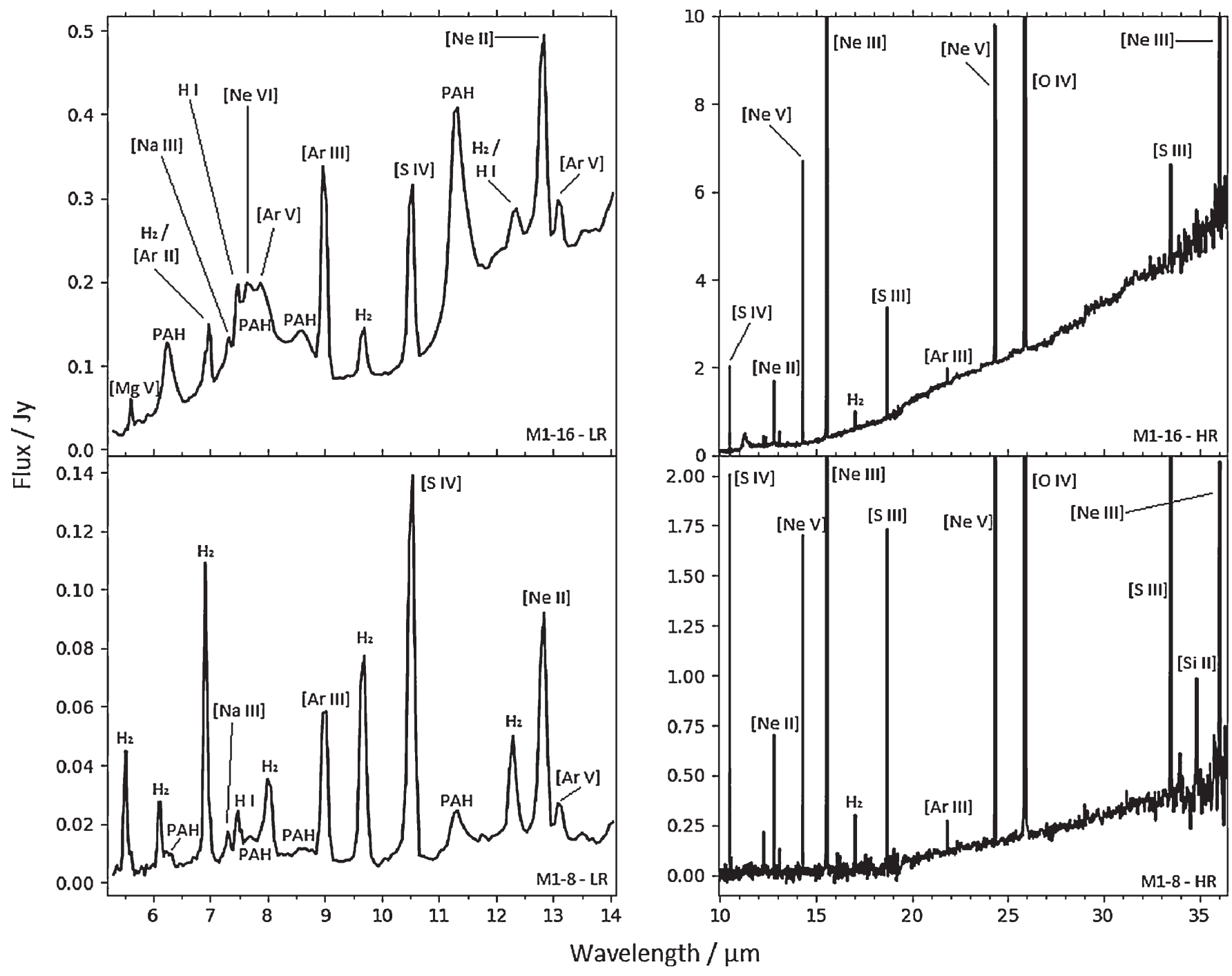

Fig. 1. Full Spitzer IRS spectrum of M1-16 (top) and M1-8 (bottom). The low resolution spectra (SL) are shown on the left, and the high resolution spectra (SH and $\mathrm{LH})$ are on the right.

Table A.2. The full low- and high-resolution spectra of two representative $\mathrm{PNe}$ in the sample, M1-16 and M1-8, are shown in Fig. 1.

\section{Analysis}

\subsection{Ionic abundances}

The ionic abundances obtained from the spectra have been calculated using the equation

$\frac{n_{\text {ion }}}{n_{p}}=n_{e} \frac{I_{\text {ion }}}{I_{H \beta}} \frac{\lambda_{u l}}{\lambda_{H \beta}} \frac{\alpha_{H \beta}}{A_{u l}}\left(\frac{n_{u}}{n_{\text {ion }}}\right)^{-1}$,

where $n_{p}$ is the proton density, $n_{e}$ is the electron density, $I_{i o n}$ is the intensity of the ion, $\lambda_{u l}$ is the line wavelength, $\alpha_{H \beta}$ is the effective recombination coefficient for $H \beta, A_{u l}$ is the Einstein coefficient of spontaneous emission, and $n_{u} / n_{i o n}$ is the ratio of the upper level population of the transition to the entire population of the ion.

For the derivation of the neon, sulphur, and argon abundances, we have observed the emission lines for the most populated ions of these elements: $\mathrm{Ne}^{+}, \mathrm{Ne}^{2+}, \mathrm{Ne}^{4+}, \mathrm{S}^{2+}, \mathrm{S}^{3+}$, $\mathrm{Ar}^{+}, \mathrm{Ar}^{2+}$ and $\mathrm{Ar}^{4+}$. We complemented our IR ionic abundances with those of the missing ionisation states from optical spectra $\left(\mathrm{Ne}^{3+}, \mathrm{S}^{+}\right.$and $\left.\mathrm{Ar}^{3+}\right)$ in the literature in order to avoid or reduce the need for ICFs. These values were primarily taken from Henry et al. (2010) and references included in Sterling \& Dinerstein (2008). We compared IR and optical data, so homogeneity in the slit sizes was assumed. This is a reasonable assumption as Henry et al. (2010) use data from the Apache Point Observatory (APO), for which the slit size is $2^{\prime \prime} \times 360^{\prime \prime}$, and most of the PNe they have observed from this sample are $\lesssim 4^{\prime \prime}$ in diameter, so most of the flux will have been detected from these sources. We also considered $\mathrm{S}^{4+}$, which is not directly detectable in IR or optical spectra, although it is only expected to contribute to the overall sulphur abundance for PNe with high IP values. Where there are no flux values available for any particular line in a PN, we applied correction factors (see Sect. 3.5).

\subsection{Line flux measurements}

Flux values for the fine structure ionic emission lines with $\mathrm{a} \geqslant 3 \sigma$ detection were determined by applying Gaussian fits through the 
ISAP line fitting programme (Sturm et al. 1998) in SMART. The raw $F(\lambda)$ values were calculated for each line in each of the two observing positions (nods), which typically agreed to within $10 \%$. These were then averaged; in the few cases where there were any remaining low-level glitches obstructing a particular line, these were discarded in favour of the flux value from the nod without glitches. The associated uncertainties were propagated from those calculated from the individual flux measurements within each nod, unless the difference in flux between the two nods was greater than the assumed uncertainties. In this latter case, we considered the flux difference to be more representative of the uncertainty. The data were then corrected for extinction using the extinction law from Fluks et al. (1994). Table A.3 shows all values for the extinction corrected intensities, $I(\lambda)$. Upper limits of $3 \sigma$ or more were calculated for emission lines of the most important ions of neon, sulphur, and argon when there were no clear detections.

\section{3. $H \beta$ intensity}

In the wavelength range of Spitzer IRS we observed the emission features of several recombination transitions of atomic hydrogen $(\mathrm{HI})$, the strongest of which were observed at $7.5 \mu \mathrm{m}$ and $12.4 \mu \mathrm{m}$. Both of these emission lines account for at least two transitions; the H I 6-5, 8-6, 11-7 and 17-8 lines are blended around $7.5 \mu \mathrm{m}$ ( $\mathrm{HI} 6-5$ is the strongest of these transitions, contributing $74.43 \%$ of the total flux) whereas the H I 7-6 and 11-8 transitions both contribute to the emission line at $12.4 \mu \mathrm{m}$ (H I 7-6 provides $89.08 \%$ of this flux). We applied the Balmer decrement to obtain values of $I(H \beta)$ from Hummer \& Storey (1987), interpolated to account for the electron density and temperature values of our PNe. When both of these IR emission features were observed, their $I(H \beta)$ values agreed by up to $\sim 25 \%$, and an average was taken.

Our calculated $I(H \beta)$ values are shown in Table A.4 alongside the $F(H \beta)$ and $I(H \beta)$ values from $4861 \AA$ optical line measurements and the extinction coefficients, $C(H \beta)$, all taken from literature. In the four cases when neither of the two recombination lines were observed in a spectrum, we applied the Fluks extinction law (Fluks et al. 1994) to these literature $F(H \beta)$ values. Our values agree with those in literature mostly to within a factor of two (more for K3-90 and M1-7). In these situations we favour our IR values, as the $\mathrm{HI}$ lines are measured in the same spectra as the ionic emission that we have derived. Another advantage of using these lines to determine $F(H \beta)$ is that the extinction corrections are far smaller than those from optical wavelengths; $A_{\lambda}<4.6$ from the use of the $4861 \AA$ $H \beta$ line, whereas from the IR recombination lines we find that $A_{\lambda}<0.2$.

\subsection{Electron densities and temperatures}

Both $n_{e}$ and $T_{e}$ are needed to determine abundance values; $n_{e}$ is a direct component of their calculation (see Eq. (1)) whereas $T_{e}$ designates the statistical populations of the excited electronic states present within the ion. These are listed in Tables A.5 and A.6 respectively, and the transition probabilities and collision strengths used in calculating these values are shown in Table A.7. These values were taken from TIPbase, part of the IRON project (Hummer et al. 1993).

Infrared lines originate from electronic transitions close to the ground state. Therefore, by analysing the ratio of $I(\lambda)$ values for two transitions of the same ion, we were able to obtain $n_{e}$ values that are mostly independent of temperature. Of the line flux ratios available from our spectra, we favoured those of the [S III] 18.7/33.5 $\mu \mathrm{m}$ transitions as both of these lines are easy to measure in high resolution spectra and frequently seen together. While other line ratios were available in some PNe (e.g. [Ne III], [Ne V]), they were either detected in fewer of the PNe in our sample, or were detected at noisy wavelength regions. For instance, the [Ne III] line at $36.0 \mu \mathrm{m}$ is found at the upper wavelength region of the $\mathrm{LH}$ module, which is highly susceptible to noise above $\sim 35 \mu \mathrm{m}$. The 14.3 and $24.3 \mu \mathrm{m}$ lines of [Ne V] were only observed in 12 of the $23 \mathrm{PNe}$ in the sample, though the associated density values agree well with those of [S III]. Uncertainties averaged $\sim 20 \%$ for values of $n_{e}>1000 \mathrm{~cm}^{-3}$, though this becomes larger for the few sources where $n_{e}<1000 \mathrm{~cm}^{-3}$. In the four cases where the two [S III] lines were not directly measurable and any other line ratios were either not observed or affected by noise, we used values given in the literature from the [S II] $6716 \AA$ / $6731 \AA$ line intensity ratio. We applied the mean value derived from the [S III] line ratios in our sample of $n_{e}=3700 \mathrm{~cm}^{-3}$ for K3-69 and Y-C 2-5 as these lines were not observed in these $\mathrm{PNe}$ and there were no $n_{e}$ values given in literature. In these cases, while the uncertainty in density is high, the abundances are little affected, with neon and argon showing little change at the density extremes, and sulphur being affected by $20 \%$ at most. All density values are shown in Table A.5, with the uncertainties reflecting those of the [S III] 18.7/33.5 $\mu \mathrm{m}$ ratios. We note that for K3-90, Henry et al. (2010) apply the high density limit to estimate $n_{e}$ despite having intensity values for the [S II] $6716 \AA$ and $6731 \AA$ lines. This is due to the two values having high uncertainties. A density of $n_{e}=800 \mathrm{~cm}^{-3}$ would have been calculated with these values, which is almost within the error margins of our IR [S III] line ratio.

Measurements of $T_{e}$ require electronic transitions with large differences in energy. For this study, we relied on temperatures calculated from optical line flux values based on the transition ratios of the [N II], [O II], [O III], [S II] and [S III] lines calculated from the literature. As no $T_{e}$ values could be found in the literature for K3-65 and K3-69, for these two PNe we adopted the average value of $T_{e}=11900 \mathrm{~K}$. All of these values can be found in Table A.6.

\subsection{Elemental abundances}

One of the main advantages of analysing spectra at infrared wavelengths is that the main ionisation lines of neon, sulphur, and argon can be observed. From these lines, we have measured the ionic abundances of $\mathrm{Ne}^{+}, \mathrm{Ne}^{2+}, \mathrm{Ne}^{4+}, \mathrm{S}^{2+}, \mathrm{S}^{3+}, \mathrm{Ar}^{+}, \mathrm{Ar}^{2+}$ and $\mathrm{Ar}^{4+}$. We complemented these data with the ionic abundances of $\mathrm{S}^{+}$and $\mathrm{Ar}^{3+}$ measured by Henry et al. (2010) from optical spectra, hence fewer corrections are required in determining their elemental abundances. We accounted for $\mathrm{Ne}^{3+}$ in sources with observable $\mathrm{Ne}^{4+}$ emission, and we considered $\mathrm{S}^{4+}$ in sources with $\mathrm{O}^{3+}$, which has a greater IP $(47.22 \mathrm{eV}$ and $54.94 \mathrm{eV}$, respectively).

We corrected for these missing ionic abundances with ICFs. ICFs can either be determined empirically (e.g. Surendiranath et al. 2004; Pottasch \& Surendiranath 2005), by considering lines with similar IP values (e.g. Peimbert \& Costero 1969), or from photoionisation models (e.g. Natta et al. 1980; Kingsburgh \& Barlow 1994; Kwitter \& Henry 2001; Delgado-Inglada et al. 2014). In many cases, argon and particularly sulphur are highlighted as being complicated to correct for, as the low IPs of higher ionisation states may lead to their greater contributions towards the overall elemental abundances. Many variants 
of the ICFs for these elements have been given in literature (e.g. Kingsburgh \& Barlow 1994; Thuan et al. 1995; Kwitter \& Henry 2001) with significant disagreement between some of them (Vermeij \& van der Hulst 2002).

To account for the abundances of missing ionisation states, we complemented our IR values with optical abundances derived by Henry et al. (2010) where possible. To correct for $\mathrm{S}^{+}$and $\mathrm{Ar}^{3+}$ when these values are not available, we calculated the percentage contributions of these ions towards their respective elemental abundances for the $\mathrm{PNe}$ in our sample with these ionic abundances and applied the mean values as ICFs. In each case, the minimum and maximum values were taken as the uncertainty limits. We also applied this method to account for $\mathrm{Ar}^{+}$ in three sources for which the $7.0 \mu \mathrm{m}$ line intensity cannot be measured. For $\mathrm{Ne}^{3+}$ and $\mathrm{S}^{4+}$, we considered the range of contributions of these ions to their respective elemental abundances as given by Bernard-Salas et al. (2008), who calculated these from the analysis of the PN sample of Pottasch \& Bernard-Salas (2006) and also from the Galactic PN models of Surendiranath et al. (2004) and Pottasch \& Surendiranath (2005). Each of these ranges are shown in Table A.8. We note that we only applied ICFs correcting for these missing ionic states in the PNe for which we observed other ions with greater or similar IP values.

Tables A.9-A.11 give the ionic and elemental abundance values for $\mathrm{Ne}, \mathrm{S}$ and Ar respectively, with the empirical ICFs applied. ICFs can be uncertain, so we also compared the resulting abundances calculated with our empirical ICFs with those calculated using well-established ICFs from the literature, such as those of Kingsburgh \& Barlow (1994), Kwitter \& Henry (2001) and Delgado-Inglada et al. (2014). In all cases, we applied our ionic abundances. For the equations given in this section, we applied the notation $\mathrm{A}(\mathrm{X})=\mathrm{ICF}\left(\mathrm{X}^{m+}+\mathrm{X}^{n+}\right) \times\left(\mathrm{X}^{m+}+\mathrm{X}^{n+}\right) / \mathrm{H}$, where $\mathrm{A}(\mathrm{X})$ is the elemental abundance of $\mathrm{X}$. Table A.12 shows the supplementary data used in the following calculations from optical abundance studies. We note that some ICF equations from other studies use ionic ratios that are particularly sensitive to the electron density and temperature, such as those involving $\mathrm{O}^{+}$and $\mathrm{O}^{2+}$. Hence, in cases where the [S III] ion ratio does not adequately account for the electron density of an important ion for the ICF calculation, or the uncertainties in $T_{e}$ are large due to the dispersion of values over several studies, there may be additional uncertainty in the abundances calculated using these ICFs.

\subsubsection{Neon}

At mid-IR wavelengths, lines of $\mathrm{Ne}^{+}, \mathrm{Ne}^{2+}, \mathrm{Ne}^{4+}$ and $\mathrm{Ne}^{5+}$ can be measured, though $\mathrm{Ne}^{3+}$ is best observed in the optical and near-UV regions, respectively at $\sim 4720 \AA$ and $\sim 2424 \AA$. Unfortunately, no literature values exist for the abundance of $\mathrm{Ne}^{3+}$ in any of the PNe in the anti-centre sample.

The photoionisation model of Kingsburgh \& Barlow (1994), also used by Kwitter \& Henry (2001), used the following ICFs:

$\mathrm{ICF}\left(\mathrm{Ne}^{2+}+\mathrm{Ne}^{4+}\right)=1.5$,

$\mathrm{ICF}\left(\mathrm{Ne}^{2+}\right)=\frac{\mathrm{O}}{\mathrm{O}^{2+}}$.

However, the abundances of sources with weak radiation fields are underestimated due to the disregard of the $\mathrm{Ne}^{+}$ionic contribution to the total neon abundance (e.g. Tsamis et al. 2013). This problem is also observed in the ionic abundances of several sources in our sample of anti-centre $\mathrm{PNe}$, in which $\mathrm{Ne}^{+}$sometimes contributes more to the overall elemental abundance than $\mathrm{Ne}^{2+}$. These include K3-66, M1-6 and M1-14 (see Table A.9).

Recently, Delgado-Inglada et al. (2014) produced a newer set of ICF models to account for parameters such as effective temperatures and stellar luminosities. For $\mathrm{Ne} / \mathrm{H}$ corrections, they apply:

$\mathrm{ICF}\left(\mathrm{Ne}^{2+}+\mathrm{Ne}^{4+}\right)=\left(1.31+12.68 v^{2.57}\right)^{0.27}$

where $v=\mathrm{He}^{2+} /\left(\mathrm{He}^{+}+\mathrm{He}^{2+}\right)$. However, the requirement for $\mathrm{Ne}^{4+}$ limits the usability of this correction. They also state that the ICFs will overestimate the neon abundances unless $0.4 \lesssim v \lesssim 0.6$. The $\mathrm{He}^{+}$and $\mathrm{He}^{2+}$ abundances given for 12 of the $23 \mathrm{PNe}$ in the anti-centre sample from Henry et al. (2010) give $v$ values outside this range, ten of these sources having values of $v<0.2$ and 5 of these with $v<0.005$. PNe with very low $v$ values have small $\mathrm{He}^{2+}$ ionic abundances, which typically indicates low-ionisation sources with little or no $\mathrm{Ne}^{4+}$ emission. However, this is not true for Y-C 2-5, which has a relatively large $v(0.61)$ but no observable $\mathrm{Ne}^{4+}$ emission in its Spitzer IRS spectrum.

Table A.13 shows a comparison between the neon abundances calculated with both our empirical ICFs and the wellestablished ICFs of Kingsburgh \& Barlow (1994) and DelgadoInglada et al. (2014). There is good agreement in almost all cases between the two sets of values, though the disregard of $\mathrm{Ne}^{+}$leads to major underestimates from the Kingsburgh \& Barlow (1994) model for M1-6 and M1-14. For Y-C 2-5, the applied ICF of 2.58 leads to a much greater abundance than predicted empirically. However, as the mid-IR spectrum of this PN shows the strong emission line of $\mathrm{Ne}^{2+}$ but not those of $\mathrm{Ne}^{+}$or $\mathrm{Ne}^{4+}$, it is possible that the $\mathrm{Ne}^{3+}$ for which we are correcting may give a significant contribution to the neon abundance. The fact that we see large amounts of $\mathrm{He}^{2+}$ compared to $\mathrm{He}^{+}$in this source shows that the radiation field in Y-C 2-5 is greater than $54.4 \mathrm{eV}$ (the IP of $\mathrm{He}^{2+}$ ), and as $\mathrm{Ne}^{3+}$ ionises at $63.5 \mathrm{eV}$, a large ICF may be required.

\subsubsection{Sulphur}

As ionisation models are typically applied to optical spectra, it is normal to only see corrections for $\mathrm{S}^{3+}$ from $\mathrm{S}^{+}$and $\mathrm{S}^{2+}$ ionic abundance measurements. In these studies, the low IP of $\mathrm{S}^{2+}$ $(34.8 \mathrm{eV})$ has always been taken as an indication that larger ionic states are likely to be present in $\mathrm{PNe}$, and the similar IP of $\mathrm{O}^{+}$ $(35.1 \mathrm{eV})$ is generally considered in obtaining an ICF for sulphur. Dinerstein (1980) carried out an IR spectroscopic survey of $12 \mathrm{PNe}$ and found that the commonly used $\mathrm{ICF}$ of $\mathrm{O} / \mathrm{O}^{+}$can overpredict the measured abundances of $\mathrm{S}^{3+}$. However, the presence of even greater ionisation states must also be considered.

Based on models of H II regions from Stasińska (1978), the ICF for sulphur from optical spectra was calculated by Barker (1980) to be

$\operatorname{ICF}\left(\mathrm{S}^{+}+\mathrm{S}^{2+}\right)=\left[1-\left(1-\frac{\mathrm{O}^{+}}{\mathrm{O}}\right)^{\alpha}\right]^{-1 / \alpha}$

where $\alpha=3$, though subsequent studies argued that $\alpha=2$ (French 1981) or $2 \leqslant \alpha \leqslant 3$ (Garnett 1989) better represented the sulphur abundances. Kingsburgh \& Barlow (1994) also used this equation, with $\alpha=3$.

A different method of determining the ICF for sulphur was calculated by Kwitter \& Henry (2001), who considered newer 
atomic data and incorporated the charge exchange rates into the ICF values. They used the equation

$\mathrm{ICF}\left(\mathrm{S}^{+}+\mathrm{S}^{2+}\right)=\exp \left[-0.017+0.18 \beta-0.11 \beta^{2}+0.072 \beta^{3}\right]$

where $\beta=\log \left(\mathrm{O} / \mathrm{O}^{+}\right)$.

The models of Delgado-Inglada et al. (2014) calculate ICFs for the $\mathrm{S} / \mathrm{O}$ ratio, and multiply by the $\mathrm{O} / \mathrm{H}$ abundance:

$\log \mathrm{ICF}\left(\left(\mathrm{S}^{+}+\mathrm{S}^{2+}\right) / \mathrm{O}^{+}\right)=\frac{-0.02-0.03 \omega-2.31 \omega^{2}+2.19 \omega^{3}}{0.69+2.09 \omega-2.69 \omega^{2}}$,

$\log \mathrm{ICF}\left(\mathrm{O}^{+}+\mathrm{O}^{2+}\right)=\frac{0.08 v+0.006 v^{2}}{0.34-0.27 v}$

where $v$ is as defined in Eq. (4) and $\omega=\mathrm{O}^{2+} /\left(\mathrm{O}^{+}+\mathrm{O}^{2+}\right)$. In all cases, $\omega>0.5$ and for K3-67, M2-2 and Y-C 2-5, $\omega>0.95$.

The sulphur abundances calculated using these ICFs are shown in Table A.14. Again, the values calculated using empirical ICFs compare well with those from photoionisation models. ICFs obtained with Eq. (5) are often large when $\alpha=2$, and provide much greater estimates than those of the compared studies. However, the agreement is greatly improved when $\alpha=3$. The uncertainties in the ICF calculated from Kwitter \& Henry (2001) are larger due to their propagation, but the ICFs themselves are smaller, with most of them having values of $\leqslant 1.35$. The only exception to this is K3-90, which has a stronger radiation field than the others $\left(\mathrm{S}^{3+} / \mathrm{S}^{2+}=5.4\right)$. Despite these uncertainties, the $\mathrm{S} / \mathrm{H}$ values calculated from Eq. (6) show a good agreement with the other values. The abundances calculated using ICFs from Delgado-Inglada et al. (2014) are similar in that their uncertainties are relatively high, though in almost all cases they show better agreement with the abundances calculated using empirically determined ICFs than those from Kwitter \& Henry (2001).

\subsubsection{Argon}

In optical spectra, the $\mathrm{Ar}^{2+}, \mathrm{Ar}^{3+}$ and $\mathrm{Ar}^{4+}$ ions can all be observed. The most abundant of these ions is thought to be $\mathrm{Ar}^{2+}$, though this largely depends on the radiation field of the source.

Argon abundances calculated by Kingsburgh \& Barlow (1994) applied the following ICFs.

$\mathrm{ICF}\left(\mathrm{Ar}^{2+}+\mathrm{Ar}^{3+}+\mathrm{Ar}^{4+}\right)=\frac{1}{1-\left(\mathrm{N}^{+} / \mathrm{N}\right)}$,

$\operatorname{ICF}\left(\mathrm{Ar}^{2+}\right)=1.87 \pm 0.41$

Kwitter \& Henry (2001) built upon Eq. (9) by considering the $\mathrm{ICF}$ when only the $\mathrm{Ar}^{2+}$ and $\mathrm{Ar}^{3+}$ ionic states could be observed:

$\mathrm{ICF}\left(\mathrm{Ar}^{2+}+\mathrm{Ar}^{3+}\right)=\frac{1}{1-\left(\mathrm{N}^{+} / \mathrm{N}\right)} \times \frac{\mathrm{He}^{+}+\mathrm{He}^{2+}}{\mathrm{He}^{+}}$.

Delgado-Inglada et al. (2014) calculated their abundances in terms of $\mathrm{Ar} / \mathrm{O}$, before multiplying by the $\mathrm{O} / \mathrm{H}$ abundance calculated with the ICF from Eq. (8):

$\log \operatorname{ICF}\left(\frac{\mathrm{Ar}^{2+}}{\mathrm{O}^{+}+\mathrm{O}^{2+}}\right)=\frac{0.03 \omega}{0.4-0.3 \omega}-0.05 \quad(0.5<\omega<0.95)$.
Table A.15 shows a comparison between the $\mathrm{Ar} / \mathrm{H}$ abundances using ICFs from all studies. In general, the empirical ICFs agree well with those from each of the three comparative studies. The uncertainties in the abundances calculated from the ICFs of Delgado-Inglada et al. (2014) are large, particularly for PNe with strong ionisation fields $(\omega \gtrsim 0.95)$. This is likely due to the ICF only requiring the ionic abundance of $\mathrm{Ar}^{2+}$.

Overall, the abundances calculated with empirically determined ICFs in all three considered elements compare well with those using ICFs from the literature. The main advantage of using the empirical method is that there is no need for large ICFs due to the number of ionisation states for which we have data. While much larger ICFs are applied in the literature to account for missing ionisation states that contribute more to the elemental abundances, the resulting values relate well to those calculated empirically in which some or all of these missing ionisation states have been observed.

\subsection{Galactocentric distances}

Distances to Galactic PNe are known to be notoriously difficult to measure owing to the variation of bolometric luminosity and effective temperature values within the sources. We considered distances relative to the Galactic centre and exclusively observed $\mathrm{PNe}$ towards the anti-centre, which somewhat reduces the errors relative to those of their respective heliocentric distances $\left(R_{h}\right)$.

We converted from $R_{h}$ to $R_{g}$ using the following equation:

$$
\begin{aligned}
\left|\vec{R}_{g}\right|=\left(\left[R_{h} \cos (b) \cos (l)-R_{\odot}\right]^{2}+R_{h}^{2} \cos ^{2}(b)\right. & \sin ^{2}(l) \\
& \left.+R_{h}^{2} \sin ^{2}(b)\right)^{0.5}
\end{aligned}
$$

where we take $R_{\odot}=8.0 \pm 0.5 \mathrm{kpc}$.

Table A.1 shows the distance values used for each PN. In this paper, we primarily adopt values from Frew et al. (2016) as measured through statistical means, though where possible we prioritised the use of directly determined distances from Giammanco et al. (2011). Table A.16 shows the $R_{h}$ values of the PNe in the anti-centre sample with distance values given by Giammanco et al. (2011), alongside those from Frew et al. (2016). While there are strong disagreements in most cases, the abundances towards the anti-centre remain lower than elsewhere in the Galactic disk. The choice of data set will not affect the overall conclusions as each case presents a similar level of dispersion around the overall gradient, part of which comes from uncertainties in the distance measurements.

The uncertainties quoted by Frew et al. (2016) are estimated to be $\sim 20-30 \%$, which seem small for statistical values, though larger uncertainties will not affect the outcomes of our discussion.

\section{Discussion}

\subsection{Comparison of abundances with literature}

In Tables A.9-A.11, we have compared the elemental abundances of neon, sulphur, and argon with those available from literature. We accounted for average uncertainties of $17.5 \%$ in neon abundances for the missing $\mathrm{Ne}^{3+}$ ionisation state, $25 \%$ in sulphur abundances for $\mathrm{S}^{+}$and $\mathrm{S}^{4+}$, and $30 \%$ in argon for $\mathrm{Ar}^{3+}$ and occasionally $\mathrm{Ar}^{+}$. We used the sample of Henry et al. (2010) as our main comparison, as this is a recent study involving 12 of our $23 \mathrm{PNe}$, though we also considered the abundances shown within the study of Sterling \& Dinerstein (2008). These all involved the use of optical spectra. 

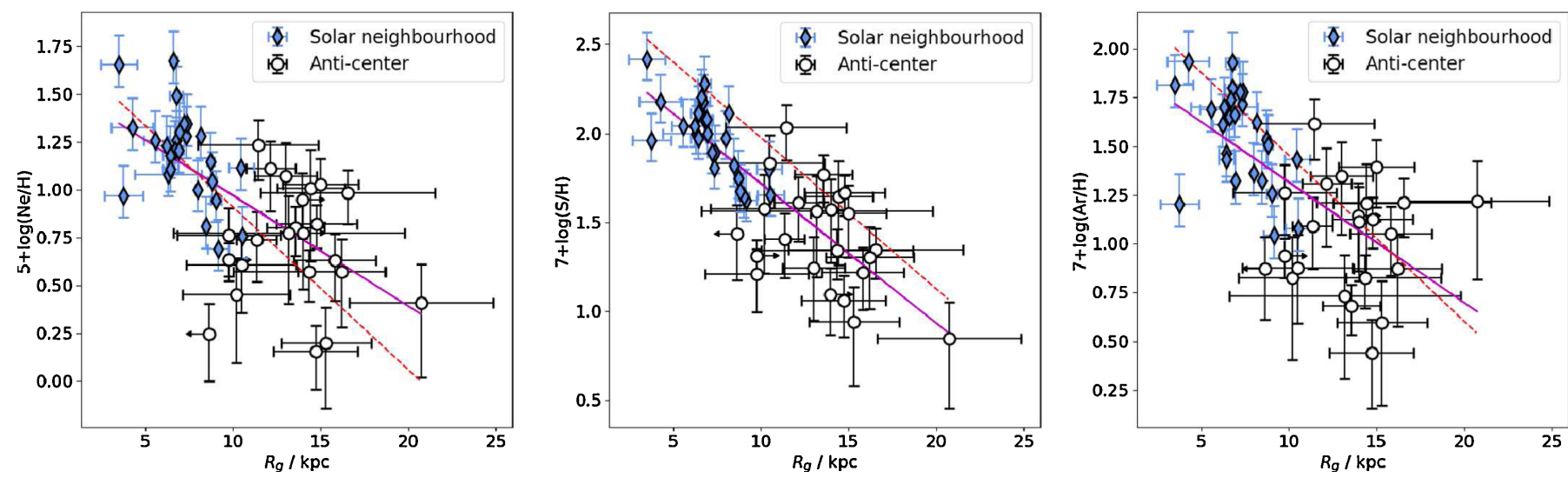

Fig. 2. Abundance gradients of neon, sulphur, and argon in the Milky Way. The dashed lines represent the oxygen abundance gradient from within the Galactic disk with a slope of $-0.085 \mathrm{dex} / \mathrm{kpc}$ (Pottasch \& Bernard-Salas 2006), passing through the solar value at $8.0 \mathrm{kpc}$ (Asplund et al. 2005). The solid lines represent the line of best fit in each plot, with gradients of $-0.058 \pm 0.021,-0.079 \pm 0.012$ and $-0.062 \pm 0.023$ dex/kpc respectively.

In most cases, there is good agreement between all sets of abundances. Where we have lower abundance values than in literature, this likely comes from over-estimated ICFs from optical studies; for example, the neon abundance of K3-90 from literature (Table A.9) has an ICF of 23.8 (from Eq. (3)) applied to an uncorrected value relatively similar to ours (Henry et al. 2010), resulting in our elemental abundances disagreeing with theirs by a factor of eight. The neon ICF was high because the oxygen ICF was comparatively large (22.3), which was calculated from the ratio of $\left(\mathrm{He}^{+}+\mathrm{He}^{2+}\right) / \mathrm{He}^{+}$abundances (Kwitter \& Henry 2001). Kingsburgh \& Barlow (1994) give the same oxygen ICF to the power of $2 / 3$, which would have decreased the ICF from 22.3 to 7.9 and the neon abundance to $(4.7 \pm 2.6) \times 10^{-5}$. This is narrowly within the uncertainty range of our $\mathrm{Ne} / \mathrm{H}$ value $\left((1.8 \pm 0.8) \times 10^{-5}\right)$.

Conversely, our neon abundance of M4-18 is a factor of 20 greater than that given in literature by De Marco \& Crowther (1999). Their abundance was calculated using the $12.8 \mu \mathrm{m}$ [Ne II] line flux from Aitken \& Roche (1982) $\left(3.6 \times 10^{-12} \mathrm{erg} \mathrm{cm}^{-2} \mathrm{~s}^{-1}\right)$, which compares well with our value, $3.3 \times 10^{-12} \mathrm{erg} \mathrm{cm}^{-2} \mathrm{~s}^{-1}$. However, the value of $\log I(H \beta)$ used by De Marco \& Crowther (1999) is lower than ours ( -11.44 and -11.24 , respectively), accounting for a discrepancy of factor 1.6. We have used $I(H \beta)$ and $C(H \beta)$ values from Henry et al. (2010), but we were unable to directly compare our neon abundances for this source as these authors were unable to observe the dominant $\mathrm{Ne}^{+}$ion, and $\mathrm{Ne}^{2+}$ was barely observable, if at all (they gave its ionic abundance with a $90 \%$ uncertainty).

The source M1-1 also shows relatively large discrepancies between our abundance and those given in literature for neon and argon. By considering its high ionisation field, from which we can observe $\mathrm{Ne}^{5+}$, we expect that $\mathrm{Ne}^{3+}$ and $\mathrm{Ar}^{3+}$ will contribute significantly to their overall abundances. Aller et al. (1986) estimate the abundances of $\mathrm{Ne}^{2+}, \mathrm{Ne}^{3+}$ and $\mathrm{Ne}^{4+}$ in $\mathrm{M} 1-1$, obtaining values of a factor of approximately three lower than our values, with $\mathrm{Ne}^{+3}$ contributing $40 \%$ of the total of those three ions (taken from measurements of the $2424 \AA$ [Ne IV] line). They also estimate $\mathrm{Ar}^{3+}$ to contribute $31 \%$ of the total argon abundance. ICFs of 1.12 and 2.1, for neon and argon respectively, are applied to their overall abundances. Even so, there are still significant discrepancies between our sets of abundance values. The abundances of the anti-centre PNe agree well with those from literature. For the few cases in which there are discrepancies between these values, they are likely to come from the larger ICFs used within the literature.

\subsection{The abundance gradient}

Figure 2 shows the abundances of neon, sulphur, and argon plotted against $R_{g}$ for the PNe in the anti-centre sample and for those of Pottasch \& Bernard-Salas (2006) from the solar neighbourhood. Both of these samples were analysed and reduced in a similar way.

The abundances are lower than those of the Galactic bulge and the solar neighbourhood, and are consistent with a continuation of the metallicity gradient up to $R_{g} \sim 20 \mathrm{kpc}$, albeit with a large dispersion within the data. However, when analysing the neon and argon anti-centre data separately from the solar neighbourhood data, there is no clear correlation, with Pearson correlation coefficients $\left(R_{P C C}\right)$ of $\sim-0.05$ in each case. Hence, we cannot discern with our data whether there is a gradient in the anti-centre $\left(R_{g}>10 \mathrm{kpc}\right)$. For sulphur, the anti-centre data show $R_{P C C}=-0.45$ with a corresponding $p$-value of 0.032 , showing that there is a slight negative correlation in the anti-centre that is statistically significant. Together with the solar neighbourhood data, $R_{P C C}=-0.64,-0.82$ and -0.66 for neon, sulphur, and argon respectively.

Table A.17 compares the radial metallicity gradients from a selection of studies over multiple wavebands, including PNe, H II regions, young B-type stars and Cepheid variables. Our analysis includes studies of neon, sulphur, and argon gradients. We also considered oxygen; even though its abundance changes over the course of stellar evolution in PNe, Pottasch \& Bernard-Salas (2006) suggested that the oxygen gradient of sources that had not undergone hot bottom burning was identical to those of the other three elements from their PN sample. Our metallicity gradient slopes compare well with those from most other studies, though ours have greater uncertainties which arise from the dispersion at greater $R_{g}$, primarily due to the uncertainties in the distance measurements. Despite this, the slopes calculated from the PN studies of Maciel \& Quireza (1999), Pottasch \& Bernard-Salas (2006) and this work are typically steeper than those of other source types. Our metallicity gradients are consistent with a continuation at high $R_{g}$, though the slopes suggest an eventual flattening or steepening with distance is possible, particularly for neon and argon. While some of the studies we considered rule out flattening as a possibility, the studies of Andrievsky et al. (2002a,b,c) and Luck et al. (2003), analysing Cepheid variables, find discontinuities in the abundance gradient with $R_{g}$. They show that the gradient is seen to be steeper in regions closer to the Galactic centre $\left(R_{g} \sim 4-6 \mathrm{kpc}\right)$ 

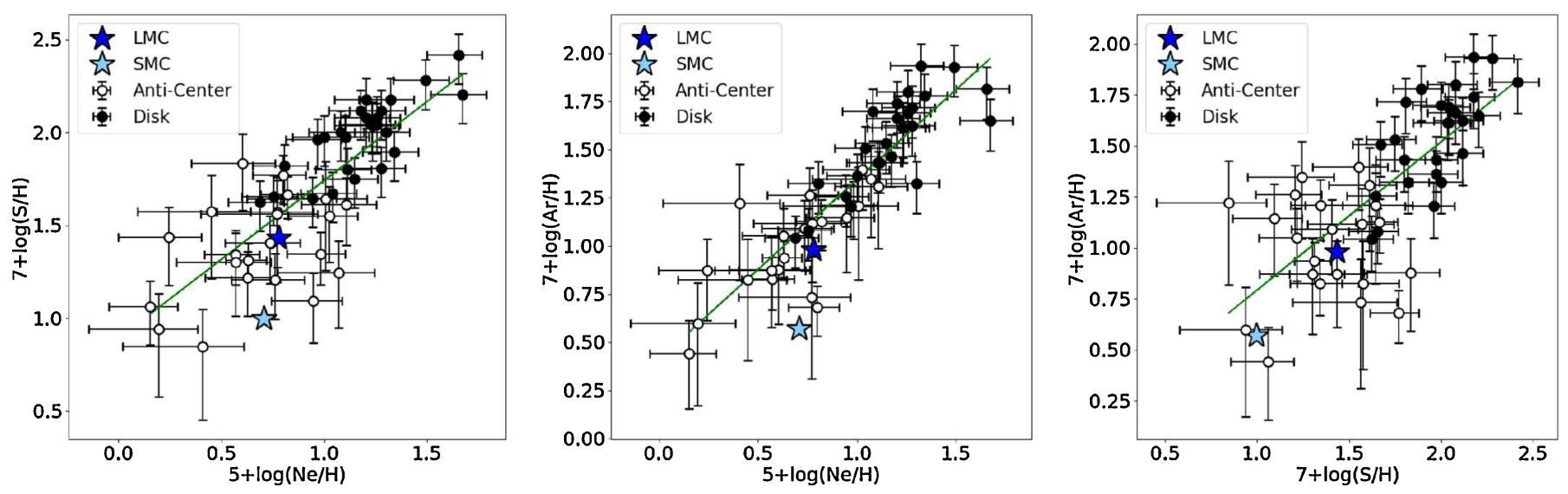

Fig. 3. Plots comparing the neon, sulphur, and argon abundances. The Galactic disk sample was analysed by Pottasch \& Bernard-Salas (2006). The average abundances for the LMC and SMC are also shown, with those of neon and sulphur obtained from Bernard-Salas et al. (2008) and argon from Leisy \& Dennefeld (2006).

and towards the anti-centre $\left(R_{g} \sim 10-15 \mathrm{kpc}\right)$ for 25 different elements, including oxygen and sulphur. Several studies that analyse sources beyond $\sim 15 \mathrm{kpc}$ (e.g. Rolleston et al. 2000; Lemasle et al. 2013; Fernández-Martín et al. 2017; this work) show steeper gradients than those found in most other studies. While this appears to agree with the findings of Andrievsky et al. (2002a,b,c), there are relatively few sources at these distances in each named study, hence the effect of the large distance sources on the respective gradients is likely to be minimal. By factoring in the large uncertainties in distance measurements for most sources in these samples, we do not find that the abundance gradients steepen with $R_{g}$ in the direction of the anti-centre.

Analysis of the time evolution of the Galactic abundance gradient from this PN sample is also difficult, due to the large uncertainties in abundances and Galactocentric distances. Our data are consistent with a continuation of the gradient at large distances, so there is no suggestion that the inner and outer disks of the Milky Way evolved separately (e.g. Stanghellini \& Haywood 2010; Kubryk et al. 2015).

\section{3. $\alpha$-process elements}

In the evolution of low- to intermediate-mass stars, the abundances of elements heavier than carbon are generally not affected, except for those formed during the slow neutroncapture process (known as the $s$-process) which can occur during the AGB phase (e.g. Lugaro et al. 2012). As a result, the abundances of neon, sulphur, and argon should trace each other. Figure 3 shows that there is good agreement between these abundances, hence proving that they do trace each other well, though it is clear that the plot of sulphur against argon shows a greater dispersion. This could be explained by the need to account for two ions for argon $\left(\mathrm{Ar}^{+}\right.$and $\left.\mathrm{Ar}^{3+}\right)$, for which $\mathrm{Ar}^{3+}$ can be the dominantion.

Included on these plots are the abundances of $\mathrm{Ne}, \mathrm{S}$ and $\mathrm{Ar}$ from the Magellanic Clouds. The mean abundance values for neon and sulphur were taken from an IR Spitzer sample of PNe from Bernard-Salas et al. (2008), and those for argon were taken from an optical PN sample from Leisy \& Dennefeld (2006). The anti-centre sample shows abundances scattered around the LMC metallicity for each of the three elements, with few reaching values below those of the SMC.

The sulphur anomaly is the term coined by Henry et al. (2004), used to describe the observed sulphur abundances in
PNe being lower than those of $\mathrm{H}$ II regions at the same metallicity (see also e.g. Henry et al. 2012). It was originally suggested that this could be explained by the lack of measured emission lines of ionisation states of $\mathrm{S}^{3+}$ and above in optical spectra and the need to account for them, particularly as $\mathrm{S}^{3+}$ can be a key stage of ionisation for sulphur. The sulphur anomaly has been seen in multiple galaxies; García-Rojas et al. (2016) observe this anomaly from four H II regions with abundances greater than most of the thirteen PNe in their sample from NGC 6822. Shaw et al. (2012) find the anomaly in the Magellanic Clouds from a combination of IR, optical and UV data, and Shingles \& Karakas (2013) find the anomaly in the Milky Way from the PN sample of Pottasch \& Bernard-Salas (2010), also from spectra in the same wavebands, compared to the ISM trend of H II regions and blue compact galaxies from the optical sample of Milingo et al. (2010).

In Fig. 4, we compare the sulphur abundances of PNe in the Galactic anti-centre and solar neighbourhood (Pottasch \& Bernard-Salas 2006) with the sulphur abundances in two samples of Galactic HII regions, one derived from IR data (Martín-Hernández et al. 2002) and the other from optical data (Fernández-Martín et al. 2017). Both of these samples cover a similar range of Galactocentric distance values to the anti-centre PNe.

The IR H II region data from Martín-Hernández et al. (2002) agree well with the PN abundances, inferring that the sulphur anomaly is not observed from these data. However, their H II region abundances disagree with the interstellar and solar values of sulphur by a factor of approximately two to four. Based on this, they argue that their abundances are underestimated by up to a factor of four, which they ascribe to uncertainties in their $n_{e}$ and $T_{e}$ values, with the lack of $\mathrm{S}^{+}$abundance values from their IR data from ISO accounting for a further $\sim 15 \%$ discrepancy. We note that since the release of the paper of Martín-Hernández et al. (2002), the most widely used solar sulphur abundance value from the literature shows a decrease of $\sim 20 \%$ from the value they used (Snow \& Witt 1996; Asplund et al. 2009), though both the abundances of the anti-centre PNe and the HII regions remain low in comparison.

Comparing the infrared PN abundances with the optical H II region abundance data of Fernández-Martín et al. (2017), with Galactocentric distances of 11-17 kpc, shows a clear discrepancy between the two sets of data. The PN abundances are lower than those of the HII regions by a factor of approximately two, as 


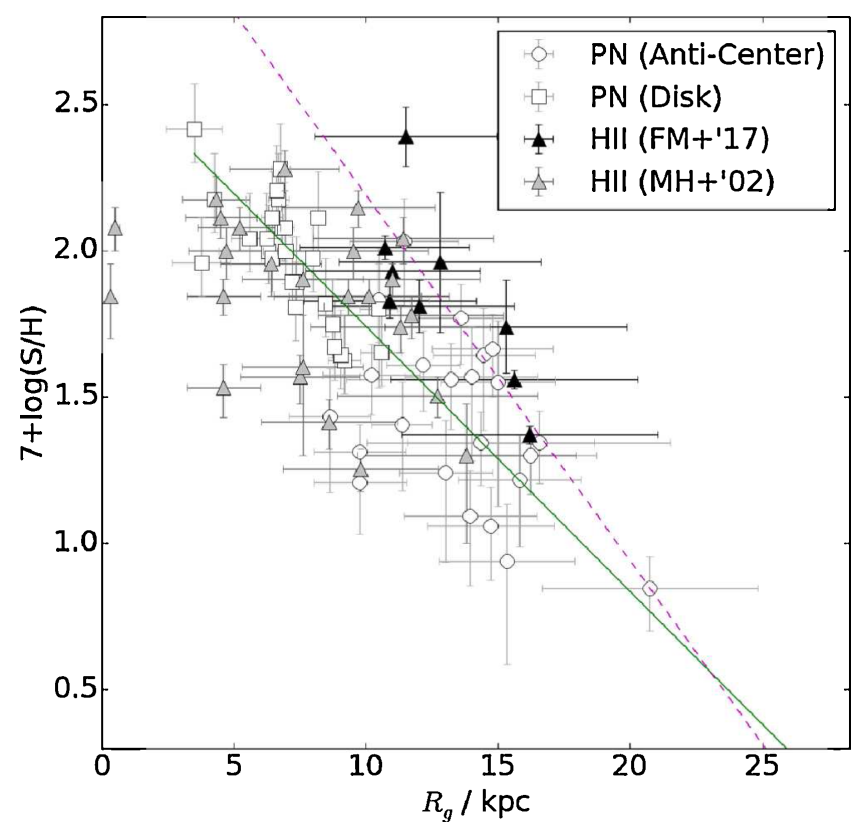

Fig. 4. Sulphur abundances of our $\mathrm{PNe}$ alongside samples of H II regions from Fernández-Martín et al. (2017) (FM+'17) and Martín-Hernández et al. (2002) (MH+'02), and solar neighbourhood PNe from Pottasch \& Bernard-Salas (2006). The solid line is the line of best fit for PN abundances. The dashed line represents that for the H II regions of the FM+'17 sample.

shown by the two lines of best fit in Fig. 4. In this case, we clearly observe the sulphur anomaly.

\section{Summary}

We have presented an infrared spectroscopic study of $23 \mathrm{PNe}$ in the Galactic anti-centre with $R_{g}$ values of 8-21 kpc using Spitzer IRS to determine the abundances of neon, sulphur, and argon in a region that is a priori assumed to be metal-poor.

We have calculated the abundances in two ways: using empirically calculated ICFs from a combination of IR and optical data, and using more well-established ICFs from the literature. We find that the two methods produce similar results; the empirical ICFs consider a wider range of ionic states and are therefore small in value. We find that the abundances of neon, sulphur, and argon are lower in the anti-centre than those in the solar neighbourhood. The metallicity gradients of these elements seem to continue beyond $R_{g}=10 \mathrm{kpc}$ despite a large spread of data values. The abundances of the $\alpha$-process elements trace each other well, though there is a slightly larger dispersion between those of sulphur and argon.

Spitzer IRS has enabled the study of abundances from observations of PNe in the bulge, disk and halo of the Milky Way, as well as in nearby galaxies (primarily the Magellanic Clouds) at infrared wavelengths. With its greater sensitivity, the James Webb Space Telescope (JWST) will be able to continue to obtain spectra for PNe as far as the Local Group of galaxies, enabling us to carry out abundance studies over a wider range of parameter space. In addition, JWST will be able to spatially resolve PNe in the Milky Way, allowing us to investigate how the gas and dust content varies within these nebulae.

Acknowledgements. We thank the anonymous referee for their feedback and comments. This research has made use of the NASA/IPAC Infrared Science Archive, which is operated by the Jet Propulsion Laboratory, California Institute of Technology, under contract with the National Aeronautics and Space Administration.
This work has been supported by a Marie Curie FP7 CIG grant under project number 630861 (FEASTFUL).

\section{References}

Acker, A., Raytchev, B., Stenholm, B., \& Tylenda, R. 1991, A\&AS, 90, 89 Aitken, D. K., \& Roche, P. F. 1982, MNRAS, 200, 217

Aksaker, N., Yerli, S. K., Kızıloğlu, Ü., \& Atalay, B. 2015, PASA, 32, 3

Aller, L. H., \& Czyzak, S. J. 1983, ApJS, 51, 211

Aller, L. H., \& Keyes, C. D. 1987, ApJS, 65, 405

Aller, L. H., Keyes, C. D., \& Feibelman, W. A. 1986, Proc. Natl. Acad. Sci., 83, 2777

Anders, F. Chiappini, C., Minchev, I., et al. 2017, A\&A, 600, A70

Andrievsky, S. M., Bersier, D., Kovtyukh, V. V., et al. 2002a, A\&A, 384, 140

Andrievsky, S. M., Kovtyukh, V. V., Luck, R. E., et al. 2002b, A\&A, 381, 32

Andrievsky, S. M., Kovtyukh, V. V., Luck, R. E., et al. 2002c, A\&A, 392, 491

Asplund, M., Grevesse, N., \& Sauval, A. J. 2005, in Cosmic Abundances as Records of Stellar Evolution and Nucleosynthesis, eds. T. G. Barnes III, \& F. N. Bash, ASP Conf. Ser. (San Fransisco: Astronomical Society of the Pacific), 336, 25

Asplund, M., Grevesse, N., Sauval, A. J., \& Scott, P. 2009, ARA\&A, 47, 481

Barker, T. 1980, ApJ, 240, 99

Bernard-Salas, J. 2003, PhD Thesis, University of Groningen, The Netherlands

Bernard-Salas, J., Pottasch, S. R., Gutenkunst, S., Morris, P. W., \& Houck, J. R. 2008, ApJ, 672, 274

Böhm-Vitense, E. 1958, Z. Astrophys., 46, 108

Butler, K., \& Zeippen, C. J. 1994, A\&AS, 108, 1

Carrasco, L., Serrano, A., \& Costero, R. 1983, Rev. Mex. Astron. Astrofis., 8, 187

Chiappini, C., Matteucci, F., \& Gratton, R. 1997, ApJ, 477, 765

Chiappini, C., Matteucci, F., \& Romano, D. 2001, ApJ, 554, 1044

Condon, J. J., \& Kaplan, D. L. 1998, ApJS, 117, 361

Costa, R. D. D., Chiappini, C., Maciel, W. J., \& de Freitas Pacheco, J. A. 1996, A\&AS, 116, 249

Costa, R. D. D., Uchida, M. M. M., \& Maciel, W. J. 2004, A\&A, 423, 199

Cuisinier, F., Acker, A., \& Koeppen, J. 1996, A\&A, 307, 215

de Freitas Pacheco, J. A., Maciel, W. J., Costa, R. D. D., \& Barbuy, B. 1991, A\&A, 250, 159

De Marco, O., \& Crowther, P. A. 1999, MNRAS, 306, 931

Delgado-Inglada, G., Morisset, C., \& Stasińska, G. 2014, MNRAS, 440, 536

Delgado-Inglada, G., Rodríguez, M., Peimbert, M., Stasińska, G., \& Morisset, C. 2015, MNRAS, 449, 1797

Dinerstein, H. L. 1980, ApJ, 237, 486

Esteban, C., García-Rojas, J., \& Pérez-Mesa, V. 2015, MNRAS, 452, 1553

Esteban, C., Fang, X., García-Rojas, J., \& Toribio San Cipriano, L. 2017, MNRAS, 471, 987

Fernández-Martín, A., Pérez-Montero, E., Vílchez, J. M., \& Mampaso, A. 2017, A\&A, 597, A84

Fitzsimmons, A., Dufton, P. L., \& Rolleston, W. R. J. 1992, MNRAS, 259, 489

Fluks, M. A., Plez, B., The, P. S., et al. 1994, A\&AS, 105, 311

French, H. B. 1981, ApJ, 246, 434

Frew, D. J., Bojičić, I. S., \& Parker, Q. A. 2013, MNRAS, 431, 2

Frew, D. J., Parker, Q. A., \& Bojičić, I. S. 2016, MNRAS, 455, 1459

Friel, E. D. 1995, ARA\&A, 33, 381

Galavis, M. E., Mendoza, C., \& Zeippen, C. J. 1995, A\&AS, 111, 347

Galavis, M. E., Mendoza, C., \& Zeippen, C. J. 1997, A\&AS, 123, 159

Galavis, M. E., Mendoza, C., \& Zeippen, C. J. 1998, A\&AS, 131, 499

García-Hernández, D. A., Ventura, P., Delgado-Inglada, G., et al. 2016, MNRAS, 458, L118

García-Rojas, J., Peña, M., Flores-Durán, S., \& Hernández-Martínez, L. 2016, A\&A, 586, A59

Garnett, D. R. 1989, ApJ, 345, 282

Genovali, K., Lemasle, B., da Silva, R., et al. 2015, A\&A, 580, A17

Giammanco, C., Sale, S. E., Corradi, R. L. M., et al. 2011, A\&A, 525, A58

Goodrich, R. W., \& Dahari, O. 1985, ApJ, 289, 342

Griffin, D. C., \& Badnell, N. R. 2000, J. Phys. B At. Mol. Phys., 33, 4389

Griffin, D. C., Mitnik, D. M., \& Badnell, N. R. 2001, J. Phys. B At. Mol. Phys., 34,4401

Henry, R. B. C., Kwitter, K. B., \& Balick, B. 2004, AJ, 127, 2284

Henry, R. B. C., Kwitter, K. B., Jaskot, A. E., et al. 2010, ApJ, 724, 748

Henry, R. B. C., Speck, A., Karakas, A. I., Ferland, G. J., \& Maguire, M. 2012, ApJ, 749, 61

Herwig, F., Bloecker, T., Schoenberner, D., \& El Eid, M. 1997, A\&A, 324 L81

Higdon, S. J. U., Devost, D., Higdon, J. L., et al. 2004, PASP, 116, 975

Houck, J. R., Roellig, T. L., van Cleve, J., et al. 2004, ApJS, 154, 18

Hummer, D. G., \& Storey, P. J. 1987, MNRAS, 224, 801

Hummer, D. G., Berrington, K. A., Eissner, W., et al. 1993, A\&A, 279, 298 
Johnson, C. T., Kingston, A. E., \& Dufton, P. L. 1986, MNRAS, 220, 155

Karakas, A. I., \& Lattanzio, J. C. 2014, PASA, 31, 30

Kingsburgh, R. L., \& Barlow, M. J. 1994, MNRAS, 271, 257

Koeppen, J., Acker, A., \& Stenholm, B. 1991, A\&A, 248, 197

Kubryk, M., Prantzos, N., \& Athanassoula, E. 2015, A\&A, 580, A127

Kwitter, K. B., \& Henry, R. B. C. 2001, ApJ, 562, 804

Lebouteiller, V., Bernard-Salas, J., Sloan, G. C., \& Barry, D. J. 2010, PASP, 122, 231

Leisy, P., \& Dennefeld, M. 2006, A\&A, 456, 451

Lemasle, B., François, P., Genovali, K., et al. 2013, A\&A, 558, A31

Luck, R. E., \& Lambert, D. L. 2011, AJ, 142, 136

Luck, R. E., Gieren, W. P., Andrievsky, S. M., et al. 2003, A\&A, 401, 939

Lugaro, M., Karakas, A. I., Stancliffe, R. J., \& Rijs, C. 2012, ApJ, 747, 2

Maciel, W. J., \& Quireza, C. 1999, A\&A, 345, 629

Maciel, W. J., Costa, R. D. D., \& Uchida, M. M. M. 2003, A\&A, 397, 667

Maciel, W. J., Costa, R. D. D., \& Cavichia, O. 2015, Rev. Mex. Astron. Astrofis., 51,165

Magrini, L., Coccato, L., Stanghellini, L., Casasola, V., \& Galli, D. 2016, A\&A, 588, A91

Magrini, L., Randich, S., Kordopatis, G., et al. 2017, A\&A, 603, A2

Marigo, P. 2001, A\&A, 370, 194

Marigo, P., Bernard-Salas, J., Pottasch, S. R., Tielens, A. G. G. M., \& Wesselius, P. R. 2003, A\&A, 409, 619

Martín-Hernández, N. L., Peeters, E., Morisset, C., et al. 2002, A\&A, 381, 606

Mendoza, C. 1983, in Planetary Nebulae, ed. D. R. Flower, IAU Symp., 103, 143

Mendoza, C., \& Zeippen, C. J. 1982, MNRAS, 199, 1025

Mendoza, C., \& Zeippen, C. J. 1983, MNRAS, 202, 981

Milingo, J. B., Henry, R. B. C., \& Kwitter, K. B. 2002, ApJS, 138, 285

Milingo, J. B., Kwitter, K. B., Henry, R. B. C., \& Souza, S. P. 2010, ApJ, 711, 619

Minchev, I., Chiappini, C., \& Martig, M. 2013, A\&A, 558, A9

Minchev, I., Famaey, B., Quillen, A. C., et al. 2012, A\&A, 548, A127

Minchev, I., Chiappini, C., \& Martig, M. 2014, A\&A, 572, A92

Mitnik, D. M., Griffin, D. C., \& Badnell, N. R. 2001, J. Phys. B At. Mol. Phys. 34, 4455

Natta, A., Preite-Martinez, A., \& Panagia, N. 1980, ApJ, 242, 596

Peimbert, M., \& Costero, R. 1969, Boletin de los Observatorios Tonantzintla y Tacubaya, 5,3

Peimbert, M., Luridiana, V., \& Torres-Peimbert, S. 1995, Rev. Mex. Astron. Astrofis., 31, 147
Pelan, J., \& Berrington, K. A. 1995, A\&AS, 110, 209

Pereira, C.-B., \& Miranda, L.-F. 2007, A\&A, 467, 1249

Perinotto, M., \& Corradi, R. L. M. 1998, A\&A, 332, 721

Phillips, J. P., \& Márquez-Lugo, R. A. 2011, Rev. Mex. Astron. Astrofis., 47, 83

Pignatari, M., Herwig, F., Hirschi, R., et al. 2016, ApJS, 225, 24

Pottasch, S. R., \& Beintema, D. A. 1999, A\&A, 347, 975

Pottasch, S. R., \& Bernard-Salas, J. 2006, A\&A, 457, 189

Pottasch, S. R., \& Bernard-Salas, J. 2010, A\&A, 517, A95

Pottasch, S. R., \& Bernard-Salas, J. 2015, A\&A, 583, A71

Pottasch, S. R., \& Surendiranath, R. 2005, A\&A, 432, 139

Rolleston, W. R. J., Smartt, S. J., Dufton, P. L., \& Ryans, R. S. I. 2000, A\&A, 363,537

Rubin, R. H., Simpson, J. P., Erickson, E. F., \& Haas, M. R. 1988, ApJ, 327, 377

Saraph, H. E., \& Storey, P. J. 1999, A\&AS, 134, 369

Sellwood, J. A., \& Binney, J. J. 2002, MNRAS, 336, 785

Shaver, P. A., McGee, R. X., Newton, L. M., Danks, A. C., \& Pottasch, S. R. 1983, MNRAS, 204, 53

Shaw, R. A., Lee, T.-H., Stanghellini, L., et al. 2012, IAU Symp., 283, 502

Shingles, L. J., \& Karakas, A. I. 2013, MNRAS, 431, 2861

Smartt, S. J. 2000, in The Evolution of the Milky Way: stars versus clusters, eds. F. Matteucci, \& F. Giovannelli, Astrophys. Space Sci. Lib. (Dordrecht: Kluwer Academic Publishers), 255, 323

Snow, T. P., \& Witt, A. N. 1996, ApJ, 468, L65

Stanghellini, L., \& Haywood, M. 2010, ApJ, 714, 1096

Stasińska, G. 1978, A\&A, 66, 257

Sterling, N. C., \& Dinerstein, H. L. 2008, ApJS, 174, 158

Sturm, E., Bauer, O. H., Brauer, J., et al. 1998, in Astronomical Data Analysis Software and Systems VII, eds. R. Albrecht, R. N. Hook, \& H. A. Bushouse ASP Conf. Ser., 154, 161

Surendiranath, R., Pottasch, S. R., \& García-Lario, P. 2004, A\&A, 421, 1051

Tamura, S., \& Shaw, R. A. 1987, PASP, 99, 1264

Thuan, T. X., Izotov, Y. I., \& Lipovetsky, V. A. 1995, ApJ, 445, 108

Tsamis, Y. G., Flores-Fajardo, N., Henney, W. J., Walsh, J. R., \& Mesa-Delgado, A. 2013, MNRAS, 430, 3406

Vera-Ciro, C., D’Onghia, E., Navarro, J., \& Abadi, M. 2014, ApJ, 794, 173

Vermeij, R., \& van der Hulst, J. M. 2002, A\&A, 391, 1081

Werner, M. W., Roellig, T. L., Low, F. J., et al. 2004, ApJS, 154, 1

Zhang, H. L., Graziani, M., \& Pradhan, A. K. 1994, A\&A, 283, 319 


\section{Appendix A: Tables}

Table A.1. 23 PNe observed in the sample.

\begin{tabular}{ccccccc}
\hline \hline Source name & Source PNG & $\begin{array}{c}\text { RA }(\mathrm{J} 2000) \\
(\mathrm{h} \mathrm{m})\end{array}$ & $\begin{array}{c}\text { Dec }(\mathrm{J} 2000) \\
(\mathrm{d} m \mathrm{~m})\end{array}$ & AORkey & $\begin{array}{c}\text { AORKey } \\
\text { off position }\end{array}$ & $\begin{array}{c}R_{g} \\
(\mathrm{kpc})\end{array}$ \\
\hline J320 & $190.3-17.7$ & 050534.32 & +104223.8 & 21946880 & 21947136 & $13.6 \pm 1.6$ \\
K3-65 & $153.7-01.4$ & 041554.53 & +484940.1 & 21947392 & 21947648 & $11.5 \pm 2.1^{*}$ \\
K3-66 & $167.4-09.1$ & 043637.23 & +333930.0 & 21947904 & 21948160 & $15.8 \pm 2.3$ \\
K3-67 & $165.5-06.5$ & 043947.93 & +364542.6 & 21948416 & 21948672 & $14.4 \pm 4.3^{\dagger}$ \\
K3-68 & $178.3-02.5$ & 053135.86 & +285841.6 & 21948928 & 21949184 & $10.2 \pm 1.8^{*}$ \\
K3-69 & $170.7+04.6$ & 054122.13 & +391508.1 & 21949440 & 21949696 & $>13.9^{*}$ \\
K3-70 & $184.6+00.6$ & 055845.34 & +251843.8 & 21949952 & 21950208 & $>14.0^{*}$ \\
K3-71 & $184.8+04.4$ & 061354.98 & +265257.0 & 21950464 & 21950720 & $10.5 \pm 2.0^{*}$ \\
K3-90 & $126.3+02.9$ & 012458.70 & +653834.7 & 21950976 & 21951232 & $<8.7^{*}$ \\
K4-48 & $201.7+02.5$ & 063955.84 & +110630.3 & 21952000 & 21952256 & $16.6 \pm 5.0^{\dagger}$ \\
M1-1 & $130.3-11.7$ & 013719.43 & +502811.6 & 21952512 & 21952768 & $14.7 \pm 2.4$ \\
M1-6 & $211.2-03.5$ & 063545.13 & -000537.5 & 21953024 & 21953280 & $9.8 \pm 1.8^{*}$ \\
M1-7 & $189.8+07.7$ & 063720.96 & +240035.4 & 21953536 & 21953792 & $14.5 \pm 1.9$ \\
M1-8 & $210.3+01.9$ & 065333.79 & +030827.0 & 21954048 & 21954304 & $12.2 \pm 1.4$ \\
M1-9 & $212.0+04.3$ & 070519.20 & +024659.5 & 21954560 & 21954816 & $16.2 \pm 2.5$ \\
M1-14 & $234.9-01.4$ & 072756.50 & -201322.8 & 21955072 & 21955328 & $11.4 \pm 1.1$ \\
M1-16 & $226.7+05.6$ & 073718.93 & -093848.0 & 21955584 & 21955840 & $13.0 \pm 1.8$ \\
M1-17 & $228.8+05.3$ & 074022.19 & -113229.9 & 21956096 & 21956352 & $14.8 \pm 2.3$ \\
M2-2 & $147.8+04.1$ & 041315.04 & +565658.1 & 21956608 & 21956864 & $>9.7 *$ \\
M3-2 & $240.3-07.6$ & 071449.92 & -275023.3 & 21957120 & 21957376 & $15.3 \pm 2.6$ \\
M4-18 & $146.7+07.6$ & 042550.85 & +600712.8 & 21957632 & 21957888 & $15.0 \pm 2.2$ \\
SaSt2-3 & $232.0+05.7$ & 074803.67 & -140740.4 & 21958144 & 21958400 & $20.8 \pm 4.1$ \\
Y-C 2-5 & $240.3+07.0$ & 081041.64 & -203132.6 & 21958656 & 21958912 & $13.2 \pm 6.6^{\ddagger}$ \\
\hline
\end{tabular}

Notes. Galactocentric distances and their errors were determined from heliocentric values determined statistically from Frew et al. (2016), assuming $R_{g, \odot}=8.0 \pm 0.5 \mathrm{kpc}$, except: * directly measured distances from Giammanco et al. (2011); ${ }^{\dagger}$ statistical distances from Phillips \& Márquez-Lugo (2011); ${ }^{\ddagger}$ statistical distance from Costa et al. (2004), from which we assume a 50\% error.

Table A.2. Aperture corrections applied to each of the line flux values.

\begin{tabular}{cccc}
\hline \hline PN & Diameter $\left(^{\prime \prime}\right)$ & SL $\rightarrow$ SH & SH $\rightarrow$ LH \\
\hline J320 & 7 & 1.40 & 1.47 \\
K3-65 & 5 & 1.20 & 1.15 \\
K3-66 & 2 & 1.05 & 1.00 \\
K3-67 & 2 & 1.00 & 1.00 \\
K3-68 & 12 & 1.50 & 2.00 \\
K3-69 & $<1$ & 1.00 & 1.00 \\
K3-70 & 2 & 1.00 & 1.05 \\
K3-71 & 3 & 1.00 & 1.00 \\
K3-90 & 10 & 1.00 & 2.00 \\
K4-48 & 2 & 1.15 & 1.08 \\
M1-1 & 5 & 1.00 & 1.50 \\
M1-6 & 4 & 1.20 & 1.12 \\
M1-7 & 11 & 1.00 & 2.20 \\
M1-8 & 18 & 1.00 & 2.00 \\
M1-9 & 3 & 1.12 & 1.07 \\
M1-14 & 5 & 1.50 & 1.30 \\
M1-16 & 3.6 & 1.38 & 1.18 \\
M1-17 & 3 & 1.18 & 1.05 \\
M2-2 & 7 & 2.30 & 2.00 \\
M3-2 & 8 & 1.00 & 1.00 \\
M4-18 & 4 & 1.12 & 1.00 \\
SaSt2-3 & $<1$ & 1.00 & 1.00 \\
Y-C 2-5 & 8 & 1.00 & 1.70 \\
\hline
\end{tabular}

Notes. A value of one implies that no correction is needed. 


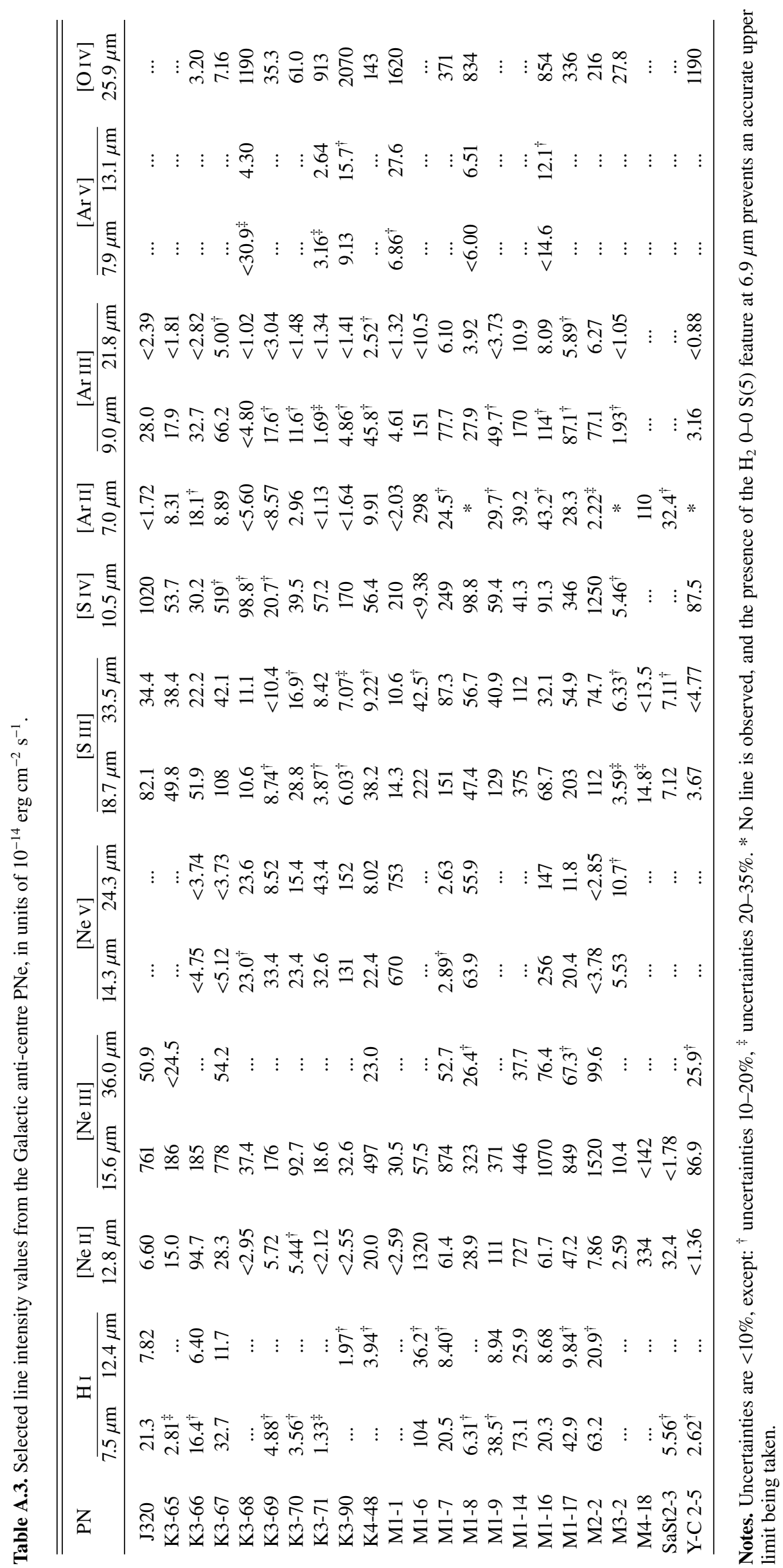


G. J. S. Pagomenos et al.: Ne, S, and Ar abundances in Galactic anti-centre PNe

Table A.4. $I(H \beta)$ values for the sample, in $\mathrm{erg}^{-2} \mathrm{~s}^{-1}$.

\begin{tabular}{ccccc}
\hline \hline PN & $C(H \beta)$ & IR $\log I(H \beta)$ & Lit. $\log F(H \beta)$ & Lit. $\log I(H \beta)$ \\
\hline J320 & 0.24 & -11.13 & $-11.63^{a},-11.39^{b}$ & -11.15 \\
K3-65 & $1.83^{\dagger}$ & -12.05 & $-14.24 .^{a}$ & -12.41 \\
K3-66 & 0.98 & -11.26 & $-12.22^{c}$ & -11.24 \\
K3-67 & 1.02 & -10.91 & $-12.13^{a},-12.07^{c}$ & -11.05 \\
K3-68 & $0.80^{\dagger}$ & $\ldots$ & $-12.90^{V}$ & -12.10 \\
K3-69 & $1.34^{\dagger}$ & -11.80 & $-13.25^{a}$ & -11.91 \\
K3-70 & 1.45 & -11.92 & $-13.54^{a},-13.59^{c}$ & -12.09 \\
K3-71 & $1.14^{\dagger}$ & -12.35 & $-13.62^{a}$ & -12.48 \\
K3-90 & 1.02 & -11.70 & $-13.40^{c}$ & -12.38 \\
K4-48 & 1.47 & -11.39 & $-12.93^{c},-12.82^{d}$ & -11.46 \\
M1-1 & $0.6^{\ddagger}$ & $\ldots$ & $-11.84^{a},-11.88^{e}$ & -11.24 \\
M1-6 & 1.57 & -10.49 & $-12.28^{a},-12.34^{c}$ & -10.77 \\
M1-7 & 0.40 & -11.15 & $-12.21^{a},-12.20^{c}$ & -11.80 \\
M1-8 & $1.1^{\ddagger}$ & -11.69 & $-13.12^{a},-12.37^{e}$ & -12.02 \\
M1-9 & 0.46 & -10.99 & $-11.66^{c},-11.73^{d}$ & -11.20 \\
M1-14 & 0.69 & -10.63 & $-11.58^{a},-12.20^{c}$ & -10.89 \\
M1-16 & 0.59 & -11.12 & $-12.80^{c},-11.99^{f}$ & -11.40 \\
M1-17 & 0.96 & -10.94 & $-12.00^{a},-11.89^{g}$ & -10.93 \\
M2-2 & 1.26 & -10.69 & $-12.22^{a},-12.63^{c}$ & -10.96 \\
M3-2 & $0.22^{\#}$ & $\ldots$ & $-13.26^{a},-12.32^{e}$ & -12.10 \\
M4-18 & 0.77 & $\ldots$ & $-12.01^{c},-12.15^{h}$ & -11.24 \\
SaSt2-3 & $0.73^{\#}$ & -11.77 & $-12.68^{i}$ & -11.95 \\
Y-C 2-5 & 0.00 & -12.07 & $-12.65^{c},-12.26^{d}$ & -12.26 \\
\hline
\end{tabular}

Notes. High errors. $C(H \beta)$ obtained from Henry et al. (2010) except: ${ }^{\dagger}$ from Giammanco et al. (2011); ${ }^{\ddagger}$ from Condon \& Kaplan (1998); ${ }^{*}$ from Frew et al. (2013).

References. ${ }^{(a)}$ Acker et al. (1991); ${ }^{(b)}$ Milingo et al. (2002); ${ }^{(c)}$ Henry et al. (2010); ${ }^{(d)}$ Cuisinier et al. (1996); ${ }^{(e)}$ Carrasco et al. (1983); ${ }^{(f)}$ Perinotto \& Corradi (1998); ${ }^{(g)}$ Costa et al. (2004); ${ }^{(h)}$ De Marco \& Crowther (1999); ${ }^{(i)}$ Pereira \& Miranda (2007); ${ }^{(V)}$ VizieR catalogue, given reference unverified. 
Table A.5. Electron density values of $\mathrm{PNe}\left(\mathrm{cm}^{-3}\right)$.

\begin{tabular}{cccc}
\hline \hline PN & $n_{e}$ (this work) & $n_{e}$ (Lit.) & Sources \\
\hline J320 & $3350 \pm 600$ & 4800 & $1,2,3,4$ \\
K3-65 & $1150 \pm 200$ & $\ldots$ & $\ldots$ \\
K3-66 & $3150 \pm 500$ & 7700 & 1,5 \\
K3-67 & $3900 \pm 550$ & 4400 & $5,6,7,8$ \\
K3-68 & $600 \pm 300$ & 500 & 6 \\
K3-69 & $3700 \pm 3000^{\dagger}$ & $\ldots$ & $\ldots$ \\
K3-70 & $2000 \pm 650$ & 2250 & $2,5,9$ \\
K3-71 & $10000 \pm 2000^{\dagger}$ & 10000 & 8 \\
K3-90 & $400 \pm 300$ & $20000^{*}$ & 5 \\
K4-48 & $8100 \pm 2250$ & 2600 & 5,10 \\
M1-1 & $1300 \pm 450$ & 4100 & 1,11 \\
M1-6 & $11450 \pm 4700$ & 8500 & 2,5 \\
M1-7 & $1900 \pm 200$ & 1050 & 2,5 \\
M1-8 & $350 \pm 150$ & 440 & 2 \\
M1-9 & $5050 \pm 900$ & 4600 & $2,5,10$ \\
M1-14 & $5450 \pm 450$ & 5400 & $2,5,12$ \\
M1-16 & $2800 \pm 550$ & 2300 & $2,5,10,13$ \\
M1-17 & $6450 \pm 300$ & 5000 & $2,9,14,15$ \\
M2-2 & $1550 \pm 300$ & 1600 & 5 \\
M3-2 & 230 & 230 & 2 \\
M4-18 & $8000 \pm 3000^{\dagger}$ & 8000 & 16,17 \\
SaSt2-3 & $600 \pm 450$ & 2400 & 9 \\
Y-C 2-5 & $3700 \pm 3000^{\ddagger}$ & $\ldots$ & $2,5,10$ \\
\hline & & &
\end{tabular}

Notes. [S III] densities used when applicable. Literature values are averaged when there are multiple sources. No uncertainty was given for the literature value of M3-2. * High density limit. ${ }^{\dagger}$ Value from literature. ${ }^{\ddagger}$ Mean $n_{e}$ value from those derived from [S III] in our sample, with the standard deviation of all other data points as the associated uncertainty.

References. (1) Aller \& Czyzak (1983); (2) Costa et al. (2004); (3) Koeppen et al. (1991); (4) Milingo et al. (2002); (5) Henry et al. (2010); (6) Aller \& Keyes (1987); (7) Kingsburgh \& Barlow (1994); (8) Tamura \& Shaw (1987); (9) Aksaker et al. (2015); (10) Cuisinier et al. (1996); (11) Aller et al. (1986); (12) Costa et al. (1996); (13) Perinotto \& Corradi (1998); (14) de Freitas Pacheco et al. (1991); (15) Peimbert et al. (1995); (16) De Marco \& Crowther (1999); (17) Goodrich \& Dahari (1985).

Table A.6. Electron temperature values of PNe as averages of literature values.

\begin{tabular}{|c|c|c|}
\hline PN & Ionic lines & $T_{e}(\mathrm{~K})$ \\
\hline $\mathrm{J} 320$ & {$[\mathrm{~N}$ II $],[\mathrm{O} \mathrm{III}]$} & $11900 \pm 2300$ \\
\hline K3-65 & & $11900 \pm 2600$ \\
\hline K3-66 & [N II], [O II], [O III], [S II], [S III] & $10800 \pm 2500$ \\
\hline K3-67 & [N II], [O II], [O III], [S III] & $14400 \pm 3200$ \\
\hline K3-68 & {$[\mathrm{N} \mathrm{II}],[\mathrm{O} \mathrm{III}]$} & $19600 \pm 2000$ \\
\hline K3-69 & & $11900 \pm 2600$ \\
\hline K3-70 & [N II], [O II], [O III], [S III] & $13700 \pm 4500$ \\
\hline K3-71 & {$[\mathrm{O}$ III $]$} & $12600 \pm 2000$ \\
\hline K3-90 & [O II $],[\mathrm{O} \mathrm{III}]$ & $12000 \pm 2500$ \\
\hline $\mathrm{K} 4-48$ & [N II], [O II $],[\mathrm{O}$ III], [S II], [S III] & $12700 \pm 2300$ \\
\hline M1-1 & [O III $]$ & $14900 \pm 1500$ \\
\hline M1-6 & [N II], [O III], [S III] & $9800 \pm 1900$ \\
\hline M1-7 & {$[\mathrm{N} \mathrm{II}],[\mathrm{O} I I],[\mathrm{O} I \mathrm{II}],[\mathrm{S} \mathrm{II}],[\mathrm{S} \mathrm{III}]$} & $10600 \pm 4200$ \\
\hline M1-8 & {$[\mathrm{N}$ II $],[\mathrm{O} \mathrm{III}]$} & $12900 \pm 1900$ \\
\hline M1-9 & [N II], [O II], [O III], I & $10800 \pm 1800$ \\
\hline M1-14 & {$[\mathrm{N}$ II $],[\mathrm{O}$ II $],[\mathrm{O}$ III $],[\mathrm{S}$ III $]$} & $10000 \pm 3700$ \\
\hline M1-16 & {$[\mathrm{N} \mathrm{II}],[\mathrm{O} \mathrm{II}],[\mathrm{O} \mathrm{III}],[\mathrm{S} \mathrm{III}]$} & $11700 \pm 3000$ \\
\hline M1-17 & {$[\mathrm{N}$ II $],[\mathrm{O} \mathrm{III}]$} & $10700 \pm 2600$ \\
\hline M2-2 & [N II], [O III], [S III] & $12500 \pm 1500$ \\
\hline M3-2 & {$[\mathrm{N}$ II $]$} & $10200 \pm 1000$ \\
\hline M4-18 & [N II], [O II], [S II] & $6100 \pm 3000$ \\
\hline SaSt2-3 & {$[\mathrm{N} \mathrm{II}]$} & $9800 \pm 1400$ \\
\hline Y-C 2-5 & [N II], [O II], [O III] & $13000 \pm 2400$ \\
\hline
\end{tabular}

Notes. K3-65 and K3-69 adopt the average temperature of the other sources due to lack of literature values; their associated uncertainties are given to be the standard deviation of all other values. Sources are the same as those given in Table A.5. 
Table A.7. Atomic data for ions shown in Table A.3.

\begin{tabular}{ccc}
\hline \hline Ion & Transition probability & Collision strength \\
\hline $\mathrm{Ne}^{+}$ & Griffin et al. (2001) & Griffin et al. (2001) \\
$\mathrm{Ne}^{2+}$ & Galavis et al. (1997) & Butler \& Zeippen (1994) \\
$\mathrm{Ne}^{4+}$ & Galavis et al. (1997) & Griffin \& Badnell (2000) \\
$\mathrm{Ne}^{5+}$ & Mendoza (1983) & Mitnik et al. (2001) \\
$\mathrm{S}^{2+}$ & Mendoza \& Zeippen (1982) & Galavis et al. (1995) \\
$\mathrm{S}^{3+}$ & Johnson et al. (1986) & Saraph \& Storey (1999) \\
$\mathrm{Ar}^{+}$ & Mendoza (1983) & Pelan \& Berrington (1995) \\
$\mathrm{Ar}^{2+}$ & Mendoza \& Zeippen (1983) & Galavis et al. (1995) \\
$\mathrm{Ar}^{4+}$ & Mendoza \& Zeippen (1982) & Galavis et al. (1995) \\
$\mathrm{O}^{3+}$ & Galavis et al. (1998) & Zhang et al. (1994) \\
\hline
\end{tabular}

Table A.8. Percentage contributions of ions that have required the use of empirically calculated ICFs towards their respective elemental abundances.

\begin{tabular}{cccc}
\hline \hline Ion & Range & Mean & Source \\
\hline $\mathrm{Ne}^{3+}$ & $2-33 \%$ & $17.5 \%$ & Bernard-Salas et al. (2008) \\
$\mathrm{S}^{+}$ & $1-20 \%$ & $10 \%$ & This work \\
$\mathrm{S}^{4+}$ & $7-23 \%$ & $15 \%$ & Bernard-Salas et al. (2008) \\
$\mathrm{Ar}^{+}$ & $1-32 \%$ & $13 \%$ & This work \\
$\mathrm{Ar}^{3+}$ & $3-46 \%$ & $26 \%$ & This work \\
\hline
\end{tabular}

Table A.9. Ionic and total abundances of neon $\left(\times 10^{-5}\right)$.

\begin{tabular}{cccccccc}
\hline \hline Source & $\begin{array}{c}{[\mathrm{N} \mathrm{II}]} \\
\mathrm{IP}=21.56 \mathrm{eV}\end{array}$ & $\begin{array}{c}{[\mathrm{N} \mathrm{III}]} \\
\mathrm{IP}=40.96 \mathrm{eV}\end{array}$ & $\begin{array}{c}{[\mathrm{N} \mathrm{V}]} \\
\mathrm{IP}=97.12 \mathrm{eV}\end{array}$ & $\begin{array}{c}{[\mathrm{N} \mathrm{VI}]} \\
\mathrm{IP}=126.21 \mathrm{eV}\end{array}$ & $\begin{array}{c}\mathrm{ICF} \\
\mathrm{Ne} / \mathrm{H}\end{array}$ & $\begin{array}{c}\mathrm{Ne} / \mathrm{H} \mathrm{lit.} \\
\times 10^{-5}\end{array}$ \\
\hline J320 & 0.109 & 6.20 & $\ldots$ & $\ldots$ & 1.00 & $6.3 \pm 1.8$ & $5.3 \pm 1.6$ \\
K3-65 & 2.45 & 14.7 & $\ldots$ & $\ldots$ & 1.00 & $17.2 \pm 5.9$ & $\ldots$ \\
K3-66 & 2.17 & 2.09 & $<0.007$ & $\ldots$ & 1.00 & $4.3 \pm 1.6$ & $4.51 \pm 1.79$ \\
K3-67 & 0.253 & 3.47 & $<0.003$ & $\ldots$ & 1.00 & $3.7 \pm 1.1$ & $3.79 \pm 0.93,4.17 \pm 1.46$ \\
K3-68 & $<0.338$ & 2.11 & 0.218 & $\ldots$ & 1.21 & $2.8 \pm 1.6$ & $\ldots$ \\
K3-69 & 0.438 & 6.69 & 0.174 & $\ldots$ & 1.21 & $8.9 \pm 3.3$ & $\ldots$ \\
K3-70 & 0.507 & 4.23 & 0.151 & $\ldots$ & 1.21 & $5.9 \pm 2.9$ & $7.01 \pm 1.74$ \\
K3-71 & $<0.569$ & 2.60 & 0.721 & $\ldots$ & 1.21 & $4.0 \pm 1.8$ & $\ldots$ \\
K3-90 & $<0.155$ & 0.952 & 0.500 & $\ldots$ & 1.21 & $1.8 \pm 0.8$ & $14.1 \pm 8.0$ \\
K4-48 & 0.573 & 7.29 & 0.051 & $\ldots$ & 1.21 & $9.6 \pm 3.0$ & $10.5 \pm 2.5$ \\
M1-1 & $<0.048$ & 0.275 & 0.886 & 0.014 & 1.21 & $1.4 \pm 0.5$ & $8.9 \pm 2.7$ \\
M1-6 & 5.65 & 0.129 & $\ldots$ & $\ldots$ & 1.00 & $5.8 \pm 2.3$ & $1.15 \pm 1.53$ \\
M1-7 & 1.06 & 7.32 & 0.003 & $\ldots$ & 1.21 & $10.2 \pm 6.0$ & $20.8 \pm 4.8$ \\
M1-8 & 1.62 & 8.73 & 0.235 & $\ldots$ & 1.21 & $12.8 \pm 5.1$ & $\ldots$ \\
M1-9 & 1.39 & 2.32 & $\ldots$ & $\ldots$ & 1.00 & $3.7 \pm 1.8$ & $4.04 \pm 1.18$ \\
M1-14 & 4.18 & 1.28 & $\ldots$ & $\ldots$ & 1.00 & $5.5 \pm 2.2$ & $1.61 \pm 0.45$ \\
M1-16 & 0.991 & 8.44 & 0.270 & 0.008 & 1.21 & $11.8 \pm 5.8$ & $10.8 \pm 2.6,7.0 \pm 2.0$ \\
M1-17 & 0.539 & 4.91 & 0.017 & $\ldots$ & 1.21 & $6.6 \pm 1.7$ & $\ldots$ \\
M2-2 & 0.045 & 4.24 & $<0.001$ & $\ldots$ & 1.00 & $4.3 \pm 1.0$ & $4.89 \pm 1.19,5.89 \pm 3.53$ \\
M3-2 & 0.426 & 0.819 & 0.052 & $\ldots$ & 1.21 & $1.6 \pm 0.9$ & $\ldots$ \\
M4-18 & 10.6 & $<2.32$ & $\ldots$ & $\ldots$ & 1.00 & $10.6 \pm 3.9$ & $0.54 \pm 0.06$ \\
SaSt2-3 & 2.56 & $<0.068$ & $\ldots$ & $\ldots$ & 1.00 & $2.6 \pm 1.5$ & $\ldots$ \\
Y-C 2-5 & $<0.188$ & 5.90 & $<0.036$ & $\ldots$ & 1.00 & $5.9 \pm 3.4$ & $\ldots$ \\
\hline & & & & & & & $\ldots$
\end{tabular}

Notes. Optical values for ionic abundances were used for ions not observable in IR spectra. ICFs applied for [Ne IV] contributions - see Sect. 3.5. Literature values from Henry et al. (2010). Literature abundances in italics are from sources given in Sterling \& Dinerstein (2008). 
Table A.10. Ionic and total abundances of sulphur $\left(\times 10^{-6}\right)$.

\begin{tabular}{|c|c|c|c|c|c|c|}
\hline Source & $\begin{array}{c}\text { [S II }] \text { lit. } \\
\mathrm{IP}=10.36 \mathrm{eV}\end{array}$ & $\begin{array}{c}{[\text { S III }]} \\
\mathrm{IP}=23.34 \mathrm{eV}\end{array}$ & $\begin{array}{c}{[\mathrm{S} \text { IV }]} \\
\mathrm{IP}=34.79 \mathrm{eV}\end{array}$ & ICF & $\begin{array}{c}\mathrm{S} / \mathrm{H} \\
\times 10^{-6}\end{array}$ & $\begin{array}{l}\text { S/H lit. } \\
\times 10^{-6}\end{array}$ \\
\hline J320 & $\ldots$ & 1.25 & 3.16 & 1.33 & $5.8 \pm 1.7$ & $14 \pm 4$ \\
\hline K3-65 & $\ldots$ & 6.64 & 1.45 & 1.33 & $10.8 \pm 3.7$ & $\ldots$ \\
\hline K3-66 & $0.15 \pm 0.10$ & 1.13 & 0.126 & 1.18 & $1.7 \pm 0.6$ & $1.70 \pm 0.55$ \\
\hline K3-67 & $0.10 \pm 0.04$ & 0.880 & 0.886 & 1.18 & $2.2 \pm 0.7$ & $2.04 \pm 0.76,5.01 \pm 1.75$ \\
\hline K3-68 & $\ldots$ & 0.899 & 1.92 & 1.33 & $3.8 \pm 2.1$ & $\ldots$ \\
\hline K3-69 & $\ldots$ & 0.629 & 0.302 & 1.33 & $1.2 \pm 0.5$ & $\ldots$ \\
\hline $\mathrm{K} 3-70$ & $0.30 \pm 0.04^{\ddagger}$ & 2.21 & 0.642 & 1.18 & $3.7 \pm 1.8$ & $3.44 \pm 1.07$ \\
\hline K3-71 & $\ldots$ & 1.37 & 3.73 & 1.33 & $6.8 \pm 3.0$ & $\ldots$ \\
\hline K3-90 & $0.36 \pm 0.15$ & 0.303 & 1.65 & 1.18 & $2.7 \pm 1.2$ & $1.86 \pm 1.42$ \\
\hline K4-48 & $0.19 \pm 0.06$ & 1.32 & 0.370 & 1.18 & $2.2 \pm 0.7$ & $1.65 \pm 0.51,1.95 \pm 0.59$ \\
\hline M1-1 & $\ldots$ & 0.207 & 0.656 & 1.33 & $1.2 \pm 0.4$ & $\cdots$ \\
\hline M1-6 & $(0.48 \pm 0.78)$ & 1.45 & $<0.011$ & 1.11 & $1.6 \pm 0.6$ & $2.66 \pm 1.29,1.91 \pm 0.57$ \\
\hline M1-7 & $0.75 \pm 0.17$ & 2.24 & 0.743 & 1.18 & $4.4 \pm 2.6$ & $3.88 \pm 1.10$ \\
\hline M1-8 & $\ldots$ & 2.17 & 0.888 & 1.33 & $4.1 \pm 1.6$ & $\cdots$ \\
\hline M1-9 & $0.16 \pm 0.10$ & 1.69 & 0.151 & 1.00 & $2.0 \pm 1.0$ & $2.10 \pm 0.64,1.29 \pm 0.39$ \\
\hline M1-14 & $0.14 \pm 0.07$ & 2.35 & 0.049 & 1.00 & $2.5 \pm 1.0$ & $2.19 \pm 0.67,0.81 \pm 0.24$ \\
\hline M1-16 & $0.24 \pm 0.07$ & 0.981 & 0.265 & 1.18 & $1.8 \pm 0.9$ & $1.55 \pm 0.46,1.80 \pm 0.50$ \\
\hline M1-17 & $0.37 \pm 0.03^{\ddagger}$ & 2.69 & 0.857 & 1.18 & $4.6 \pm 1.2$ & $9.55 \pm 2.87$ \\
\hline M2-2 & $0.01 \pm 0.00$ & 0.526 & 1.21 & 1.18 & $2.1 \pm 0.5$ & $1.10 \pm 0.51$ \\
\hline M3-2 & $\ldots$ & 0.512 & 0.141 & 1.33 & $0.87 \pm 0.49$ & $\ldots$ \\
\hline M4-18 & $2.89 \pm 0.74^{\dagger}$ & 0.665 & $\ldots$ & 1.00 & $3.6 \pm 1.6$ & $3.56 \pm 3.85,1.45 \pm 0.20$ \\
\hline SaSt2-3 & $0.24 \pm 0.04^{\ddagger}$ & 0.476 & $\ldots$ & 1.00 & $0.70 \pm 0.42$ & $\ldots$ \\
\hline Y-C 2-5 & $\ldots$ & 0.462 & 2.27 & 1.33 & $3.6 \pm 2.1$ & $\ldots$ \\
\hline
\end{tabular}

Notes. Optical values for ionic abundances were used for ions not observable in IR spectra. ICFs applied to account for [S II] and [S V] contributions - see Sect. 3.5. Literature values from Henry et al. (2010). Literature abundances in italics are from sources given in Sterling \& Dinerstein (2008). ${ }^{\dagger}$ Abundance calculated from flux data in De Marco \& Crowther (1999). ${ }^{\ddagger}$ Abundances calculated from flux data in Aksaker et al. (2015).

Table A.11. Ionic and total abundances of argon $\left(\times 10^{-7}\right)$.

\begin{tabular}{|c|c|c|c|c|c|c|c|}
\hline Source & $\begin{array}{c}{[\mathrm{Ar} \text { II }]} \\
\mathrm{IP}=15.76 \mathrm{eV}\end{array}$ & $\begin{array}{c}{[\text { Ar III }]} \\
\mathrm{IP}=27.63 \mathrm{eV}\end{array}$ & $\begin{array}{c}\text { [Ar IV] lit. } \\
\text { IP }=40.74 \mathrm{eV}\end{array}$ & $\begin{array}{c}{[\mathrm{Ar} \mathrm{V}]} \\
\mathrm{IP}=59.81 \mathrm{eV}\end{array}$ & ICF & $\begin{array}{l}\mathrm{Ar} / \mathrm{H} \\
\times 10^{-7} \\
\end{array}$ & $\begin{array}{c}\text { Ar/H lit. } \\
\times 10^{-7} \\
\end{array}$ \\
\hline J320 & $<0.180$ & 3.55 & $\ldots$ & $\ldots$ & 1.35 & $4.8 \pm 1.4$ & $9.4 \pm 2.8$ \\
\hline K3-65 & 8.45 & 22.0 & $\ldots$ & $\ldots$ & 1.35 & $41.2 \pm 14.2$ & $\ldots$ \\
\hline K3-66 & 2.66 & 5.64 & $\ldots$ & $\ldots$ & 1.35 & $11.2 \pm 4.4$ & $5.4 \pm 1.1$ \\
\hline K3-67 & 0.487 & 4.48 & $1.73 \pm 0.35$ & $\ldots$ & 1.00 & $6.7 \pm 2.0$ & $6.0 \pm 1.2,10.0 \pm 3.5$ \\
\hline K3-68 & $<3.83$ & $<3.96$ & $\ldots$ & 1.05 & 1.35 & $<6.7 \pm 4.2$ & $\ldots$ \\
\hline K3-69 & $<4.13$ & 10.3 & $\ldots$ & $\ldots$ & 1.35 & $13.9 \pm 6.7$ & $\ldots$ \\
\hline K3-70 & 1.70 & 8.17 & $3.16 \pm 1.09$ & $\ldots$ & 1.00 & $13.0 \pm 6.6$ & $14.6 \pm 3.0$ \\
\hline K3-71 & $<1.89$ & 3.59 & & 1.98 & 1.35 & $7.5 \pm 3.6$ & 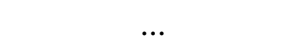 \\
\hline K3-90 & $<0.350$ & 2.23 & $3.44 \pm 0.64$ & 1.78 & 1.00 & $7.5 \pm 3.4$ & $13.3 \pm 2.4$ \\
\hline K4-48 & 1.78 & 10.2 & $4.21 \pm 0.80$ & $\ldots$ & 1.00 & $16.2 \pm 5.3$ & $19.9 \pm 3.7,5.4 \pm 4.1$ \\
\hline M1-1 & $<0.227$ & 0.632 & $\ldots$ & 1.05 & 1.64 & $2.8 \pm 1.3$ & $21.0 \pm 8.6$ \\
\hline M1-6 & 8.32 & 5.21 & $\ldots$ & $\ldots$ & 1.35 & $18.3 \pm 7.2$ & $9.1 \pm 2.3,40.7 \pm 12.2$ \\
\hline M1-7 & 2.69 & 10.2 & $3.27 \pm 0.59$ & $\ldots$ & 1.00 & $16.2 \pm 9.5$ & $36.4 \pm 6.4$ \\
\hline M1-8 & $*$ & 11.7 & $\ldots$ & 0.703 & 1.64 & $20.3 \pm 10.6$ & $\ldots$ \\
\hline M1-9 & 2.38 & 4.85 & $0.22 \pm 0.08$ & $\ldots$ & 1.00 & $7.5 \pm 3.7$ & $8.0 \pm 2.0,10.0 \pm 3.0$ \\
\hline M1-14 & 1.46 & 7.67 & $\ldots$ & $\ldots$ & 1.35 & $12.3 \pm 4.9$ & $9.5 \pm 2.0,20.4 \pm 6.1$ \\
\hline M1-16 & 4.35 & 13.9 & $3.56 \pm 0.68$ & 0.400 & 1.00 & $22.2 \pm 11.1$ & $22.2 \pm 4.0,18.0 \pm 3.0$ \\
\hline M1-17 & 2.07 & 7.83 & $\ldots$ & $\ldots$ & 1.35 & $13.4 \pm 3.9$ & $33.1 \pm 9.9$ \\
\hline M2-2 & 0.079 & 3.32 & $3.00 \pm 0.57$ & $\ldots$ & 1.35 & $8.7 \pm 2.0$ & $6.8 \pm 1.2,8.9 \pm 5.4$ \\
\hline M3-2 & $*$ & 2.41 & $\ldots$ & $\ldots$ & 1.64 & $4.0 \pm 2.5$ & $\ldots$ \\
\hline M4-18 & 24.8 & $\ldots$ & $\ldots$ & $\ldots$ & 1.00 & $24.8 \pm 9.4$ & $\ldots$ \\
\hline SaSt2-3 & 16.6 & $\ldots$ & $\ldots$ & $\ldots$ & 1.00 & $16.6 \pm 10.0$ & $\ldots$ \\
\hline Y-C 2-5 & $*$ & 3.30 & $\ldots$ & $\ldots$ & 1.64 & $5.4 \pm 3.4$ & $\ldots$ \\
\hline
\end{tabular}

Notes. Optical values for ionic abundances were used for ions not observable in IR spectra. ICFs applied to account for [Ar II] and [Ar IV] contributions - see Sect. 3.5. Literature values from Henry et al. (2010). Literature abundances in italics are from sources given in Sterling \& Dinerstein (2008). * Upper limits for $6.99 \mu \mathrm{m}[\mathrm{Ar} \mathrm{II}]$ are inaccurate due to the contribution of the $\mathrm{H}_{2} \mathrm{0}-0 \mathrm{~S}(5)$ line at $6.9 \mu \mathrm{m}$. 
G. J. S. Pagomenos et al.: Ne, S, and Ar abundances in Galactic anti-centre PNe

Table A.12. Ionic and elemental abundances from optical data in the literature, used in the calculations of neon, sulphur, and argon ICFs in Tables A.13-A.15.

\begin{tabular}{|c|c|c|c|c|c|c|c|}
\hline $\mathrm{PN}$ & $\begin{array}{c}\mathrm{He}^{+} / \mathrm{H}^{+} \\
\times 10^{-3} \\
\end{array}$ & $\begin{array}{c}\mathrm{He}^{2+} / \mathrm{H}^{+} \\
\times 10^{-3} \\
\end{array}$ & $\begin{array}{l}\mathrm{N}^{+} / \mathrm{H}^{+} \\
\times 10^{-6} \\
\end{array}$ & $\begin{array}{c}\mathrm{N} / \mathrm{H} \\
\times 10^{-6} \\
\end{array}$ & $\begin{array}{l}\mathrm{O}^{+} / \mathrm{H}^{+} \\
\times 10^{-5} \\
\end{array}$ & $\begin{array}{c}\mathrm{O}^{2+} / \mathrm{H}^{+} \\
\times 10^{-5} \\
\end{array}$ & $\begin{array}{c}\mathrm{O} / \mathrm{H} \\
\times 10^{-5} \\
\end{array}$ \\
\hline J320 & $\cdots$ & & $0.23 \pm 0.08$ & $14.8 \pm 4.4$ & $0.42 \pm 0.13$ & $25.7 \pm 7.7$ & $27.5 \pm 8.3$ \\
\hline K3-66 & $88 \pm 11$ & $0.32 \pm 0.10$ & $12.6 \pm 4.1$ & $34.1 \pm 8.1$ & $59 \pm 38$ & $10.0 \pm 2.2$ & $16.0 \pm 4.8$ \\
\hline K3-67 & $93 \pm 14$ & $0.24 \pm 0.04$ & $3.6 \pm 0.9$ & $79 \pm 23$ & $5.9 \pm 1.8$ & $12.5 \pm 3.2$ & $13.1 \pm 3.3$ \\
\hline K3-70 & $98 \pm 15$ & $21.5 \pm 3.2$ & $52 \pm 12$ & $305 \pm 81$ & $20.4 \pm 5.6$ & $7.8 \pm 2.0$ & $12.0 \pm 2.7$ \\
\hline K3-90 & $4.9 \pm 2.5$ & $105 \pm 17$ & $\ldots$ & $\ldots$ & $1.3 \pm 0.7$ & $2.7 \pm 0.7$ & $62 \pm 33$ \\
\hline $\mathrm{K} 4-48$ & $107 \pm 14$ & $17.3 \pm 2.6$ & $22.5 \pm 5.4$ & $206 \pm 50$ & $35 \pm 11$ & $24.3 \pm 5.8$ & $32.3 \pm 7.1$ \\
\hline M1-6 & $\ldots$ & & $42 \pm 20$ & $58 \pm 26$ & $241 \pm 87$ & $9.5 \pm 2.9$ & $34 \pm 11$ \\
\hline M1-7 & $110 \pm 14$ & $15.7 \pm 2.4$ & $61 \pm 14$ & $252 \pm 70$ & $115 \pm 30$ & $29.9 \pm 6.8$ & $47.3 \pm 9.2$ \\
\hline M1-9 & $104 \pm 14$ & $0.11 \pm 0.04$ & $8.8 \pm 2.8$ & $37 \pm 13$ & $47 \pm 31$ & $14.5 \pm 3.3$ & $19.2 \pm 5.0$ \\
\hline M1-14 & $96 \pm 12$ & $0.10 \pm 0.05$ & $14.0 \pm 3.9$ & $46 \pm 13$ & $89 \pm 47$ & $20.1 \pm 4.4$ & $29.0 \pm 6.9$ \\
\hline M1-16 & $105 \pm 15$ & $25.2 \pm 3.8$ & $81 \pm 20$ & $543 \pm 145$ & $50 \pm 14$ & $21.8 \pm 5.3$ & $33.2 \pm 7.2$ \\
\hline M2-2 & $104 \pm 14$ & $7.8 \pm 1.2$ & $0.36 \pm 0.12$ & $15.8 \pm 6.6$ & $4.5 \pm 0.8$ & $17.9 \pm 4.3$ & $19.8 \pm 4.7$ \\
\hline Y-C 2-5 & $39.7 \pm 5.9$ & $62.4 \pm 9.2$ & $0.15 \pm 0.05$ & $55 \pm 34$ & $0.79 \pm 0.39$ & $11.1 \pm 2.9$ & $28.6 \pm 7.7$ \\
\hline
\end{tabular}

Notes. All values have been taken from Henry et al. (2010), except for those of J320 which are from Costa et al. (2004) with an assumed 30\% uncertainty applied. Values in italics are not involved in any future calculations, and have only been included for completeness.

Table A.13. Comparison of the neon abundances and ICFs used in this study with those in which ICFs from other sources have been applied (see Sect. 3.5.1).

\begin{tabular}{|c|c|c|c|c|c|c|}
\hline \multirow[b]{2}{*}{ PN } & \multicolumn{2}{|c|}{ This work } & \multicolumn{2}{|c|}{ KB94 } & \multicolumn{2}{|c|}{ DI14 } \\
\hline & $\mathrm{Ne} / \mathrm{H} \times 10^{-5}$ & $\mathrm{ICF}(\mathrm{Ne})$ & $\mathrm{Ne} / \mathrm{H} \times 10^{-5}$ & $\mathrm{ICF}(\mathrm{Ne})$ & $\mathrm{Ne} / \mathrm{H} \times 10^{-5}$ & $\mathrm{ICF}(\mathrm{Ne})$ \\
\hline $\mathrm{J} 320$ & $6.3 \pm 1.8^{* \dagger}$ & 1.00 & $6.6 \pm 3.3^{\dagger}$ & 1.07 & $\ldots$ & $\ldots$ \\
\hline K3-66 & $4.3 \pm 1.6^{* \dagger}$ & 1.00 & $3.4 \pm 1.8^{\dagger}$ & 1.61 & $\ldots$ & $\ldots$ \\
\hline K3-67 & $3.7 \pm 1.1^{* \dagger}$ & 1.00 & $3.6 \pm 1.7^{\dagger}$ & 1.05 & $\begin{array}{l}\cdots \\
\ldots\end{array}$ & $\begin{array}{l}\cdots \\
\ldots\end{array}$ \\
\hline K3-68 & $2.8 \pm 1.6^{* \dagger \neq}$ & 1.21 & $3.5 \pm 0.7^{\dagger \neq}$ & 1.50 & $\ldots$ & $\ldots$ \\
\hline K3-69 & $8.9 \pm 3.3^{* \dagger \neq}$ & 1.21 & $10.3 \pm 3.3^{\dagger \hbar}$ & 1.50 & $\ldots$ & $\ldots$ \\
\hline K3-70 & $5.9 \pm 2.9^{* \dagger *}$ & 1.21 & $6.6 \pm 3.2^{\dagger \neq}$ & 1.50 & $4.9 \pm 2.5^{\dagger \ddagger}$ & 1.11 \\
\hline K3-71 & $4.0 \pm 1.8^{* \dagger \neq}$ & 1.21 & $5.0 \pm 2.2^{\dagger \neq}$ & 1.50 & $\ldots$ & $\ldots$ \\
\hline K3-90 & $1.8 \pm 0.8^{* \dagger *}$ & 1.21 & $2.2 \pm 0.9^{\dagger \neq}$ & 1.50 & $2.9 \pm 1.2^{\dagger \neq}$ & 1.98 \\
\hline $\mathrm{K} 4-48$ & $9.6 \pm 3.0^{* \dagger *}$ & 1.21 & $11.0 \pm 3.1^{\dagger \dagger}$ & 1.50 & $8.0 \pm 3.0^{\dagger \neq}$ & 1.09 \\
\hline M1-1 & $1.4 \pm 0.5^{* \dagger \neq \#}$ & 1.21 & $1.7 \pm 0.6^{\dagger \neq}$ & 1.50 & $\ldots$ & $\ldots$ \\
\hline M1-6 & $5.8 \pm 2.3^{* \dagger}$ & 1.00 & $0.46 \pm 0.26^{\dagger}$ & 3.54 & $\ldots$ & $\ldots$ \\
\hline M1-7 & $10.2 \pm 6.0^{* \dagger \neq}$ & 1.21 & $11.0 \pm 5.0^{\dagger \dagger}$ & 1.50 & $8.0 \pm 2.4^{\dagger \hbar}$ & 1.09 \\
\hline M1-8 & $12.8 \pm 5.1^{* \dagger \neq}$ & 1.21 & $13.4 \pm 3.2^{\dagger \neq}$ & 1.50 & $\ldots$ & $\ldots$ \\
\hline M1-9 & $3.7 \pm 1.8^{* \dagger}$ & 1.00 & $3.1 \pm 1.3^{\dagger}$ & 1.32 & $\ldots$ & $\ldots$ \\
\hline M1-14 & $5.5 \pm 2.2^{* \dagger}$ & 1.00 & $1.8 \pm 0.9^{\dagger}$ & 1.44 & $\ldots$ & $\ldots$ \\
\hline M1-16 & $11.8 \pm 5.8^{* \dagger \# \#}$ & 1.21 & $13.1 \pm 6.9^{\dagger \neq}$ & 1.50 & $9.7 \pm 3.5^{\dagger \ddagger}$ & 1.11 \\
\hline M1-17 & $6.6 \pm 1.7^{* \dagger \neq}$ & 1.21 & $7.4 \pm 2.3^{\dagger *}$ & 1.50 & $\ldots$ & $\ldots$ \\
\hline M2-2 & $4.3 \pm 1.0^{* \dagger}$ & 1.00 & $4.7 \pm 1.9^{\dagger}$ & 1.11 & $4.6 \pm 2.0^{\dagger \ddagger}$ & 1.08 \\
\hline M3-2 & $1.6 \pm 0.9^{* \dagger}$ & 1.21 & $1.3 \pm 0.5^{\dagger \neq}$ & 1.50 & $\ldots$ & $\ldots$ \\
\hline Y-C 2-5 & $5.9 \pm 3.4^{* \dagger}$ & 1.00 & $15.2 \pm 6.9^{\dagger}$ & 2.58 & $\ldots$ & $\ldots$ \\
\hline
\end{tabular}

Notes. KB94 = Kingsburgh \& Barlow (1994); DI14 = Delgado-Inglada et al. (2014). In all cases, ICFs are applied to our neon ionic abundances from Table A.9. Superscript symbols show the ions considered in the calculations: ${ }^{*}=\mathrm{Ne}^{+} ;{ }^{\dagger}=\mathrm{Ne}^{2+} ;{ }^{\sharp}=\mathrm{Ne}^{4+} ;{ }^{\#}=\mathrm{Ne}^{5+}$. M4-18 and SaSt2-3 are not included on this table as their $\mathrm{Ne}^{2+}$ abundances are upper limits. K3-65 is not included as its helium and oxygen abundances have not been found in the literature. 
Table A.14. Comparison of the sulphur abundances and ICFs used in this study with those in which ICFs from other sources have been applied (see Sect. 3.5.2).

\begin{tabular}{|c|c|c|c|c|c|c|c|c|c|c|}
\hline \multirow{3}{*}{ PN } & \multirow{2}{*}{\multicolumn{2}{|c|}{$\begin{array}{l}\text { This work } \\
\text { S/H }\end{array}$}} & \multicolumn{4}{|c|}{ B80 / KB94 } & & \\
\hline & & & \multicolumn{2}{|c|}{$\alpha=3$} & \multicolumn{2}{|c|}{$\alpha=2$} & \multirow{2}{*}{$\begin{array}{c}\mathrm{S} / \mathrm{H} \\
\times 10^{-6}\end{array}$} & \multicolumn{3}{|c|}{$\mathrm{S} / \mathrm{H}$} \\
\hline & $\times 10^{-6}$ & $\mathrm{ICF}(\mathrm{S})$ & $\mathrm{S} / \mathrm{H} \times 10^{-6}$ & $\mathrm{ICF}(\mathrm{S})$ & $\mathrm{S} / \mathrm{H} \times 10^{-6}$ & $\mathrm{ICF}(\mathrm{S})$ & & $\mathrm{ICF}(\mathrm{S})$ & $\times 10^{-6}$ & $\mathrm{ICF}(\mathrm{S})$ \\
\hline K3-66 & $1.7 \pm 0.6^{* \dagger \ddagger}$ & 1.18 & $1.4 \pm 1.0^{* \dagger}$ & 1.10 & $1.6 \pm 1.2^{* \dagger}$ & 1.29 & $\#$ & $\cdots$ & $\#$ & $\cdots$ \\
\hline K3-67 & $2.2 \pm 0.7^{* \dagger *}$ & 1.18 & $2.1 \pm 0.9^{* \dagger}$ & 1.98 & $3.6 \pm 1.5^{* \dagger}$ & 3.38 & $1.2 \pm 0.7^{* \dagger}$ & 1.22 & $1.9 \pm 1.3^{* \dagger}$ & 1.91 \\
\hline K3-70 & $3.7 \pm 1.8^{* \dagger *}$ & 1.18 & $3.3 \pm 0.6^{* \dagger}$ & 1.33 & $4.5 \pm 1.0^{* \dagger}$ & 1.79 & $2.7 \pm 1.1^{* \dagger}$ & 1.09 & $3.3 \pm 2.0^{* \dagger}$ & 1.31 \\
\hline K3-90 & $2.7 \pm 1.2^{* \dagger *}$ & 1.18 & $3.6 \pm 1.8^{* \dagger}$ & 5.39 & $10.1 \pm 5.7^{* \dagger}$ & 15.3 & $1.9 \pm 1.7^{* \dagger}$ & 2.86 & $12.4 \pm 10.2^{* \dagger}$ & 18.7 \\
\hline K4-48 & $2.2 \pm 0.7^{* \dagger \ddagger}$ & 1.18 & $2.3 \pm 0.8^{* \dagger}$ & 1.51 & $3.3 \pm 1.3^{* \dagger}$ & 2.20 & $1.7 \pm 0.9^{* \dagger}$ & 1.13 & $2.3 \pm 1.5^{* \dagger}$ & 1.52 \\
\hline M1-6 & $1.6 \pm 0.6^{\dagger}$ & 1.11 & $\#$ & ... & $\#$ & ... & $\#$ & $\cdots$ & $\#$ & \\
\hline M1-7 & $4.4 \pm 2.6^{* \dagger \neq}$ & 1.18 & $3.6 \pm 0.9^{* \dagger}$ & 1.21 & $4.6 \pm 1.3^{* \dagger}$ & 1.53 & $3.2 \pm 1.4^{* \dagger}$ & 1.07 & $3.4 \pm 1.9^{* \dagger}$ & 1.15 \\
\hline M1-9 & $2.0 \pm 1.0^{* \dagger \ddagger}$ & 1.00 & $2.2 \pm 1.5^{* \dagger}$ & 1.21 & $2.9 \pm 2.0^{* \dagger}$ & 1.53 & $\#$ & $\ldots$ & $\#$ & $\ldots$ \\
\hline M1-14 & $2.5 \pm 1.0^{* \dagger \neq}$ & 1.00 & $2.7 \pm 1.5^{* \dagger}$ & 1.14 & $3.3 \pm 1.9^{* \dagger}$ & 1.39 & $2.6 \pm 2.1^{* \dagger}$ & 1.06 & $2.6 \pm 2.4^{* \dagger}$ & 1.04 \\
\hline M1-16 & $1.8 \pm 0.9^{* \dagger *}$ & 1.18 & $1.7 \pm 0.5^{* \dagger}$ & 1.37 & $2.3 \pm 0.8^{* \dagger}$ & 1.90 & $1.3 \pm 0.6^{* \dagger}$ & 1.10 & $1.7 \pm 1.0^{* \dagger}$ & 1.38 \\
\hline M2-2 & $2.1 \pm 0.5^{* \dagger \hbar}$ & 1.18 & $1.3 \pm 0.2^{* \dagger}$ & 2.47 & $2.5 \pm 0.5^{* \dagger}$ & 4.72 & $0.72 \pm 0.25^{* \dagger}$ & 1.35 & $1.2 \pm 0.7^{* \dagger}$ & 2.28 \\
\hline
\end{tabular}

Notes. B80 = Barker (1980); KB94 = Kingsburgh \& Barlow (1994); KH01 = Kwitter \& Henry (2001); DI14 = Delgado-Inglada et al. (2014). In all cases, ICFs are applied to our sulphur ionic abundances from Table A.10. Superscript symbols show the ions considered in the calculations: ${ }^{*}=\mathrm{S}^{+}$; ${ }^{\dagger}=\mathrm{S}^{2+} ;{ }^{\ddagger}=\mathrm{S}^{3+}$. ${ }^{\#}$ Uncertainties $>100 \%$, likely due to uncertainties in the ICF and $\mathrm{S}^{+}$abundances given from Henry et al. (2010).

Table A.15. Comparison of the argon abundances and ICFs used in this study with those in which ICFs from other sources have been applied (see Sect. 3.5.3).

\begin{tabular}{|c|c|c|c|c|c|c|c|c|}
\hline \multirow[b]{2}{*}{ PN } & \multicolumn{2}{|c|}{ This work } & \multicolumn{2}{|c|}{ KB94 } & \multicolumn{2}{|c|}{ КH01 } & \multicolumn{2}{|c|}{ DI14 } \\
\hline & $\mathrm{Ar} / \mathrm{H} \times 10^{-1}$ & $\mathrm{ICF}(\mathrm{Ar})$ & $\mathrm{Ar} / \mathrm{H} \times 10^{-7}$ & $\mathrm{ICF}(\mathrm{Ar})$ & $\mathrm{Ar} / \mathrm{H} \times 10^{-1}$ & $\mathrm{ICF}(\mathrm{Ar})$ & $\mathrm{Ar} / \mathrm{H} \times 10^{-1}$ & $\overline{\mathrm{ICF}(\mathrm{Ar})}$ \\
\hline K3-66 & $11.2 \pm 4.4^{* \dagger}$ & 1.35 & $10.5 \pm 2.5^{\dagger}$ & 1.87 & $\cdots$ & $\cdots$ & $6.2 \pm 5.0^{\dagger}$ & 1.10 \\
\hline K3-67 & $6.7 \pm 2.0^{* \dagger \ddagger}$ & 1.00 & $8.4 \pm 2.0^{\dagger}$ & 1.87 & $6.5 \pm 3.3^{\dagger \ddagger}$ & 1.05 & $7.2 \pm 5.6^{\dagger}$ & 1.60 \\
\hline K3-70 & $13.0 \pm 6.6^{* \dagger \neq}$ & 1.00 & $15.3 \pm 4.1^{\dagger}$ & 1.87 & $16.7 \pm 9.5^{\dagger \star}$ & 1.47 & $11.5 \pm 10.0^{\dagger}$ & 1.40 \\
\hline K3-90 & $7.5 \pm 3.4^{* \dagger \neq \# \# ~}$ & 1.00 & $4.2 \pm 1.1^{\dagger}$ & 1.87 & $\ldots$ & ... & $\ldots$ & $\ldots$ \\
\hline K4-48 & $16.2 \pm 5.3^{* \dagger *}$ & 1.00 & $19.1 \pm 4.6^{\dagger}$ & 1.87 & $18.8 \pm 8.5^{\dagger *}$ & 1.30 & $15.3 \pm 12.3^{\dagger}$ & 1.50 \\
\hline M1-6 & $18.3 \pm 7.2^{* \dagger}$ & 1.35 & $9.7 \pm 2.3^{\dagger}$ & 1.87 & $\ldots$ & $\ldots$ & $\ldots$ & $\ldots$ \\
\hline M1-7 & $16.2 \pm 9.5^{* \dagger}$ & 1.00 & $19.1 \pm 4.6^{\dagger}$ & 1.87 & $20.3 \pm 7.2^{\dagger *}$ & 1.51 & $12.9 \pm 9.6^{\dagger}$ & 1.26 \\
\hline M1-9 & $7.5 \pm 3.7^{* \dagger \neq}$ & 1.00 & $9.1 \pm 2.4^{\dagger}$ & 1.87 & $6.7 \pm 4.0^{\dagger \star}$ & 1.31 & $5.9 \pm 4.8^{\dagger}$ & 1.21 \\
\hline M1-14 & $12.3 \pm 4.9^{* \dagger}$ & 1.35 & $14.3 \pm 3.5^{\dagger}$ & 1.87 & $11.0 \pm 8.6^{\dagger \dagger}$ & 1.44 & $8.8 \pm 8.3^{\dagger}$ & 1.14 \\
\hline M1-16 & $22.2 \pm 11.1^{* \dagger * \#}$ & 1.00 & $21.0 \pm 6.1^{\dagger \dagger \#}$ & 1.18 & $25.4 \pm 11.7^{\dagger *}$ & 1.46 & $20.2 \pm 16.2^{\dagger}$ & 1.45 \\
\hline M2-2 & $8.7 \pm 2.0^{* \dagger *}$ & 1.35 & $6.2 \pm 1.5^{\dagger}$ & 1.87 & $7.0 \pm 4.6^{\dagger \ddagger}$ & 1.10 & $5.8 \pm 4.9^{\dagger}$ & 1.74 \\
\hline Y-C 2-5 & $5.4 \pm 3.4^{\dagger}$ & 1.64 & $6.2 \pm 1.6^{\dagger}$ & 1.87 & $\ldots$ & $\ldots$ & $11.3 \pm 8.8^{\dagger}$ & 3.42 \\
\hline
\end{tabular}

Notes. KB94 = Kingsburgh \& Barlow (1994); KH01 = Kwitter \& Henry (2001); DI14 = Delgado-Inglada et al. (2014). In all cases, ICFs are applied to our argon ionic abundances from Table A.11. Superscript symbols show the ions considered in the calculations: ${ }^{*}=\mathrm{Ar}^{+} ;{ }^{\dagger}=\mathrm{Ar}^{2+} ;{ }^{\ddagger}=\mathrm{Ar}^{3+}$; ${ }^{\#}=\mathrm{Ar}^{4+}$.

Table A.16. Comparison of heliocentric distances from Giammanco et al. (2011) and Frew et al. (2016).

\begin{tabular}{ccc}
\hline \hline PN & $\begin{array}{c}R_{h} / \mathrm{kpc} \\
(\text { Giammanco+'11) }\end{array}$ & $\begin{array}{c}R_{h} / \mathrm{kpc} \\
(\text { Frew+'16) }\end{array}$ \\
\hline K3-65 & 3.7 & 13.0 \\
K3-68 & 2.2 & 7.4 \\
K3-69 & $>6.0$ & $\ldots$ \\
K3-70 & $>6.0$ & 15.8 \\
K3-71 & 2.5 & 18.2 \\
K3-90 & $<1.0$ & 7.0 \\
M1-6 & 2.0 & 5.2 \\
M2-2 & $>2.0$ & 5.2 \\
\hline
\end{tabular}


G. J. S. Pagomenos et al.: Ne, S, and Ar abundances in Galactic anti-centre PNe

Table A.17. Comparison of several abundance gradient studies which use various sources.

\begin{tabular}{|c|c|c|c|c|c|}
\hline Study & Sources & Waveband & Element & Slope (dex/kpc) & $R_{g}$ range $(\mathrm{kpc})$ \\
\hline This work & $\mathrm{PNe}$ & IR, Optical & $\begin{array}{l}\mathrm{Ne} \\
\mathrm{S} \\
\mathrm{Ar}\end{array}$ & $\begin{array}{l}-0.058 \pm 0.021 \\
-0.079 \pm 0.012 \\
-0.062 \pm 0.023\end{array}$ & $\begin{array}{l}3-21 * \\
3-21 * \\
3-21 *\end{array}$ \\
\hline Maciel \& Quireza (1999) & $\mathrm{PNe}$ & IR, Optical & $\begin{array}{c}\mathrm{O} \\
\mathrm{Ne} \\
\mathrm{S} \\
\mathrm{Ar}\end{array}$ & $\begin{array}{l}-0.058 \pm 0.007 \\
-0.036 \pm 0.010 \\
-0.077 \pm 0.011 \\
-0.051 \pm 0.010\end{array}$ & $\begin{array}{l}4-14 \\
4-14 \\
4-13 \\
4-13\end{array}$ \\
\hline Pottasch \& Bernard-Salas (2006) & $\mathrm{PNe}$ & IR, Optical, UV & $\mathrm{O}, \mathrm{Ne}, \mathrm{S}, \mathrm{Ar}$ & -0.085 & $3-11$ \\
\hline Maciel et al. (2015) & $\mathrm{PNe}$ & Multiple & $\mathrm{O}$ & $-0.025 \pm 0.006$ & $0-15$ \\
\hline Martín-Hernández et al. (2002) & H II regions & Optical & $\begin{array}{l}\mathrm{Ne} \\
\mathrm{Ar}\end{array}$ & $\begin{array}{l}-0.039 \pm 0.007 \\
-0.045 \pm 0.011\end{array}$ & $\begin{array}{l}0-14 \\
0-13\end{array}$ \\
\hline Esteban et al. (2017) & H II regions & Optical & $\mathrm{O}$ & $-0.040 \pm 0.005$ & $5-17^{\dagger}$ \\
\hline Fernández-Martín et al. (2017) & H II regions & Optical & $\begin{array}{c}\mathrm{O} \\
\mathrm{S} \\
\mathrm{Ar}\end{array}$ & $\begin{array}{l}-0.053 \pm 0.009 \\
-0.106 \pm 0.006 \\
-0.074 \pm 0.006\end{array}$ & $\begin{array}{l}11-18 \\
11-18 \\
11-18\end{array}$ \\
\hline Fitzsimmons et al. (1992) & B-type stars & Multiple & $\mathrm{O}$ & $-0.03 \pm 0.02$ & $5-14$ \\
\hline Rolleston et al. (2000) & B-type stars & Optical & $\mathrm{O}$ & $-0.067 \pm 0.008$ & $6-18$ \\
\hline Andrievsky et al. (2002b) & Cepheids & IR & $\begin{array}{l}\mathrm{O} \\
\mathrm{S}\end{array}$ & $\begin{array}{l}-0.022 \pm 0.009 \\
-0.051 \pm 0.008\end{array}$ & $\begin{array}{l}6-11 \\
6-11\end{array}$ \\
\hline Lemasle et al. (2013) & Cepheids & Optical, near-IR & S & $-0.095 \pm 0.015$ & $4-19 \ddagger$ \\
\hline Henry et al. (2004) & $\begin{array}{c}\text { PNe, H II regions, } \\
\text { B-type stars, } \\
\text { Cepheids }\end{array}$ & Multiple & $\begin{array}{c}\mathrm{O} \\
\mathrm{Ne} \\
\mathrm{S} \\
\mathrm{Ar}\end{array}$ & $\begin{array}{l}-0.037 \pm 0.008 \\
-0.044 \pm 0.014 \\
-0.048 \pm 0.010 \\
-0.030 \pm 0.010\end{array}$ & $\begin{array}{l}0-18 \\
2-14 \\
0-17 \\
2-17\end{array}$ \\
\hline
\end{tabular}

Notes. Studies with "multiple" wavebands use data from several references. * Includes data from Pottasch \& Bernard-Salas (2006); ${ }^{\dagger}$ Includes data from Esteban et al. (2015); Includes data from Luck \& Lambert (2011). 